\title{
A Sequential Auction-Bargaining Procurement Model
}

\author{
Woonghee Tim Huh Kun Soo Park \\ Industrial Engineering and Operations Research Department, Columbia University \\ 500 West 120th Street, MC 4704, New York NY 10027, USA \\ th2113@columbia.edurkp2143@columbia.edu
}

\begin{abstract}
One of the most important decisions that a firm faces in managing its supply chain is a procurement decision: selecting suitable suppliers among many potential competing sellers and reducing the purchase cost. While both auction and bargaining have been extensively studied in the literature, the research that combines both auction and bargaining is limited. In this paper, we consider a combined auction-bargaining model in a setting where a single buyer procures an indivisible good from one of many competing sellers. The procurement model that we analyze is a sequential model consisting of the auction phase followed by the bargaining phase. In the auction phase, the sellers submit bids, and the seller with the lowest bid is selected as the winning bidder. In the bargaining phase, the buyer audits the cost of the winning seller and then negotiates with him to determine the final price. For this auction-bargaining model, we find a symmetric bidding strategy equilibrium for the sellers in a closed form, which is simple to understand and closely related to the classical results in the auction and bargaining literature. We also show that the auction-bargaining model generates at least as much profit to the buyer as the standard auction or sequential bargaining model.
\end{abstract}

Keywords: Auction; Bargaining; Procurement; Single-Unit

\section{Introduction}

Since procurement costs often constitute a large portion of a firm's total operating costs Bonser and $\mathrm{Wu}[6]$, selecting suppliers with attractive prices is an important decision that a firm faces in managing its supply chain. The type of the transaction method for conducting procurement decisions varies greatly depending on the context, ranging from (i) pricing (including both static and dynamic pricing) to (ii) auctions and (iii) one-to-one bargaining (Elmaghraby et al. [16]). While the posted price mechanism is popular in business-toconsumer transactions, both auctions and bargaining are now widespread in business-tobusiness procurement (Bajari et al. [1]). Each of these three methods have been studied in the operations management literature, e.g., Federgruen and Heching [20], Chen [13], Chen et al. [14], and Nagarajan and Bassok [33], but we note that many transactions in business 
practice cannot simply be categorized as pricing, auction or bargaining, as they may have the characteristics of multiple methods. There have been a number of papers that combines features of pricing and auction, e.g., Caldentey and Vulcano [10] and Gallien and Gupta [22]. In this paper, we consider a procurement method that sequentially combine auction and bargaining, where the outcome of the auction is not final and is subject to further negotiation.

The motivation for this paper stems from our observation that the use of auctions in the procurement process often does not completely determine the final outcome of the procurement decision. Auctions perform poorly when the projects are complex and the contracts cannot be fully specified (Bajari et al. [1]). Suppliers feel that the auction format of procurement erodes their control over the final price and "commoditizes" their products (Jap [24]). However, it is quite common that a purchasing manager will solicit bids from the pool of potential suppliers (either by telephone, mail, or Internet), and based on the bids that she has received, she will decide which buyers to examine closely and to possibly negotiate with. The round of indicative bidding is valuable to the buyer in the case that the cost associated with studying a supplier as part of due diligence is high. For example, in the sale of Daewoo Moters in 1999, Ford bid in the indicative round between $\$ 5.4$ and $\$ 6.3$ billion, higher than DaimlerChrysler-Hyundai's joint bid of $\$ 4.5$ billion, and subsequently Ford was chosen as the sole bidder in the final negotiation phase (Ye [45]). In a supply chain setting, the post-auction negotiation provides the buyer and the supplier with an opportunity to learn more about the costs, knowledge and performance expectation (Dalya and Nath [15]).

Both auction and bargaining are commonly used in practice, and have been thoroughly studied in the literature. Auctions provide efficiency and simplicity in connecting the buyer to the seller with the lowest cost (Manelli and Vincent [28]), and the buyer prefers auctions when she has relatively less information about the sellers' cost (Waehrer [41]). There exists a vast amount of literature on the theoretical analysis of auctions in both the economics and the operations management literature; we refer the reader to Krishna [26] and Menezes and Monteiro [30] for a review of the auction theory and Myerson [32] for the optimal auction design. The types of auctions include: first price or second price auctions, sealed-bid or open-bid auctions, and auctions with or without a reserve price. Another common way of determining the terms of trade (e.g., supplier selection and price decision) is bargaining, which occurs between the buyer and one or many sellers (Nagarajan and Bassok [33]). According to Bajari et al. [1], bargaining was used in 45 percent of procurement decisions for the 
public-sector non-residential construction projects in Northern California between 1995 and 2000. For more references in the bargaining literature, we refer to Rubinstein [36] or Osborne and Rubinstein [35] for a general review, and $\mathrm{Wu}$ [44] for an emphasis on procurement. Examples of commonly used bargaining models include the ultimatum take-it-or-leave-it offers, and the sequential alternating offers.

While there are several papers that compare auction and bargaining in terms of minimizing the procurement cost, empirical and experimental studies yield no clear verdict. For example, Kjerstad [25] argues from an empirical study of procurement contracts of medical and surgical products that auctions do not provide significantly lower prices compared to bargaining. Bajari et al. [1] empirically analyze contracts awarded in the construction industry, and find that auctions do not perform well in some cases because of the insufficient number of bidders. In an empirical study of timber sales, Leffler et al. [27] note that conducting an auction might incur a significantly high cost to the auctioneer if she does not use professional assistance from a forestry consultant; in this case, auctions would be less preferable to bargaining. In an experimental study, Thomas and Wilson [39] compare the first price auction to multilateral bargaining between a single buyer and multiple sellers where the buyer solicits price offers by showing each seller his rival's price offers while restricting communication between sellers. They report that, with four sellers, the buyer's acquisition costs through auction and bargaining are almost the same. While the above-mentioned comparisons are with respect to cost, many papers make comparisons along other dimensions such as the quality of the product, for example, Manelli and Vincent [28], Bonaccorsi et al. [5], and Tunca and Zenios [40].

In this paper, we consider a setting where a risk-neutral profit maximizing buyer procures an indivisible product from one of many competing suppliers. We propose a model that combines auction and bargaining sequentially in two phases. The first phase is the standard auction such as the first or second price auction where one seller is selected among multiple competing sellers. At this time, while the buyer's value is public information, each seller's cost is private information unknown to other sellers and the buyer. In the second phase, the buyer bargains with the chosen seller over the final price of the product. We assume that, at the beginning of the bilateral bargaining, information regarding the seller's true cost becomes available to the buyer. This assumption is justifiable if the value and cost information can be accurately estimated (through the on-going supplier-customer relationship, the maturity of the market, or an additional investigation as a part of due-diligence), or if such information 
is equally uncertain to both parties of bargaining. (The buyer's true value has already become public in the first stage - thus, the buyer does not have any information advantage over the chosen seller.) The outcome of bargaining results in a price that is between the seller's true cost and the buyer's valuation. During the bargaining process, the buyer has the option to purchase the product at the price that is equal to the seller's winning bid in the first phase. For this combined auction-bargaining system, we study the sellers' equilibrium bidding strategy in the first phase, and also the buyer's choice of the reserve price, if allowed.

\subsection{Literature Review}

While the procurement systems that combine auction and bargaining are not uncommon in practice, the literature on the analysis of such models is rather limited (EngelbrechtWiggans and Katok [18]). Single-unit sequential auction-bargaining models with the firstphase auction and the second-phase bargaining have been studied by Bulow and Klemperer [8], Elyakime et al. [17] and Wang [43].

The seminal paper in this literature is Bulow and Klemperer [8]. They propose an auction-

bargaining sequential model, where the winning bidder is determined by an open-bid second price auction, followed by a take-it-or-leave-it offer by the auctioneer (which may be accepted or rejected by the only remaining bidder). The major result of this paper is that while the auctioneer's ability to make an ultimatum bargaining offer increases her expected profit, the amount of this increase is bounded above by the expected gain of having one additional bidder in the standard auction. (Thus, the buyer prefers having an additional seller in the auction as opposed to conducting the second phase of ultimatum bargaining.) While Bulow and Klemperer [8] use the ultimatum bargaining model with incomplete information, we are able to incorporate the relative bargaining power of the buyer and the seller in a bargaining model with complete information. We also show that their major result mentioned above may not hold if a bargaining model other than the ultimatum bargaining is used.

Elyakime et al. [17] study a single-unit sequential model, where the first phase is the first price sealed-bid auction where the auctioneer also submits a secret sealed-bid reserve price. If all the bids do not meet the reserve price, then the second-phase bargaining takes place, and the trade occurs with the auctioneer and the most-attractive bidder where the gain of trade is split equally between them. Thus, the cost and value information becomes public at the beginning of the second phase (as in our model). They present an equilibrium bidding strategy as a solution to a first-order differential equation. Numerical results indicate that 
both the auctioneer and the bidders prefer this model to the auction model that does not have the possibility of second phase bargaining.

In the model proposed by Wang [43], the buyer has a private valuation (unknown to sellers), and does not submit any bid in the auction phase. The seller with the most attractive bid becomes the winning seller. The buyer has the choice of either accepting the winning bid of the auction as the price of the product, or entering into second-phase bargaining with the winning seller. In the latter case, the winning seller's cost becomes known to the buyer while the buyer's valuation remains private, and the second-phase bargaining is modeled as a Rubinstein-style dynamic game with one-sided uncertainty. For this model, the sellers' symmetric equilibrium strategy is given as a solution to a first-order differential equation. As his model is the closest to our model, we highlight the difference between the two models. (1) While the buyer's valuation is private in his model, we model her valuation as public information. (2) Whereas the buyer in his model decides whether to continue to the second phase where the winning seller's bid no longer has any effect, the buyer in our model always continues to the bargaining phase where the winning bid remains consequential and acts as an outside option. (3) While he models the equal bargaining power between the buyer and the winning seller, we allow the possibility that the buyer may have a stronger bargaining power than the seller. (4) While he considers only the first price auction in the first phase, we consider both the first price and the second price auctions. (Our analysis later shows that the second price auction generates more profit to the buyer than the first price auction.) (5) More importantly, while there is no closed-form bidding strategy in Wang [43], we find an equilibrium bidding strategy in a closed form that is simple to analyze and intuitive to understand.

While the above papers involve only a single unit, Salmon and Wilson [37] consider an auction-bargaining sequential model with two identical units. The buyer procures one unit through an auction in the first phase, followed by a second phase where the second unit may be procured through a take-it-or-leave-it offer from the seller with the second highest bid in the auction phase. They show the nonexistence of the pure strategy for the sellers. Experimental results indicate that the buyer's profit in this model is higher than the sequential auction where the second phase is also conducted as an auction.

In all of the auction-bargaining models mentioned thus far, auction is followed by bargaining; we mention that Engelbrecht-Wiggans and Katok [18] consider a model where bargaining precedes auction. The buyer wants to procure multiple products from sellers, each capable 
of producing one unit. In the first phase, the sellers' costs have not been realized, and the buyer offers some sellers an opportunity in which they may commit to procure one unit at a price to be established later in the second-phase auction. These sellers are excluded from the second-phase auction, regardless of whether or not they have accepted the buyer's offer, and the second-phase auction is conducted as a generalization of the second price auction. In addition, the growing literature on the auctions with the buyout option ("buy it now" option) could be considered as a combined bargaining-auction model, where the buyout price acts as a take-it-or-leave-it offer before the bidders participate in the auction. It has been noted that when the bidders exhibit impatience over time, this option can increase the auctioneer's profit (Mathews [29], Budish and Takeyama [7], Hidvégi et al. [23], Caldentey and Vulcano [10], Gallien and Gupta [22]).

\subsection{Contribution}

There are a limited number of papers that combine auction and bargaining, especially compared to the vast literature on auction and on bargaining, and our paper studies a sequential auction-bargaining model that complements existing models in the literature. Our model admits a symmetric equilibrium bidding strategy in a closed form. This is our main contribution. (In all the existing auction-bargaining models, the bidders' equilibrium strategies are either simple truthtelling or unable to be expressed in a closed form.) As a result, we are able to compute the buyer's expected profit, and show that she generates higher profit in the auction-bargaining model compared to standard auction or bargaining stand-alone models. In the special case of uniform cost distributions, we show that the equilibrium strategy that we find is unique. Interestingly, the equilibrium bidding strategy in our model is closely tied to the standard results in the classical auction and bargaining literature.

As our second contribution, we study the auction-bargaining model where the buyer announces a reserve price at the beginning of the auction phase. The reserve price increases the buyer's expected profit in this model, consistent with the auction model, and we show how to find the optimal reserve price. In the auction-bargaining model, we find that the buyer sets a reserve price that is less aggressive than in the standard auction-only model. We also characterize the sellers' equilibrium bidding strategies when the buyer's reserve price is present.

Our third contribution is to compare the use of the first price auction and the second price auction during the auction phase of the model. While the expected profit of the buyer 
in a standard auction remains the same regardless of the auction format, we find that, in the sequential auction-bargaining model where the Revenue Equivalence Theorem does not apply, the expected profit of the buyer is higher when the second price auction is used in the auction phase than the first price auction. Thus, a risk-neutral profit-maximizing buyer prefers the second price auction-bargaining model to the first price auction-bargaining model.

The organization of this paper is as follows. In Section 2, we describe the auctionbargaining model in detail. In Section 3, we summarize some preliminary results in the auction and bargaining theory for further analysis. In Section 4, we study the auction-bargaining model, and in particular develop the sellers' symmetric equilibrium bidding strategy. Our analysis includes the use of the second price auction, the first price auction, and the buyer's reserve price. We consider extensions and variants of the auction-bargaining model in Section 5, and we conclude in Section 6. All the proofs are in the appendix of this paper.

\section{Model Description}

Suppose that a buyer (e.g., a manufacturer) wants to procure an indivisible product from one of $n+1$ potential sellers (e.g., suppliers), where $n \geq 1$. The products offered by these sellers are identical, and the sellers are indexed by $i=1,2, \ldots, n+1$. Each seller $i$ 's opportunity cost of the product is drawn from a random variable $C_{i}$, and we assume that the distributions of $C_{i}$ 's are independent and identical, having the support of $[\underline{c}, \bar{c}]$, where $\underline{c} \geq 0$ and $\bar{c}<\infty$. Let $F(\cdot)$ and $f(\cdot)$ denote the cumulative distribution function and the probability density function of each $C_{i}$, respectively. We assume that the ex post $\operatorname{cost} c_{i}$ of seller $i$ is initially private information, but the distribution function $F(\cdot)$ is public information. It is convenient to define a random variable $Y$, which denotes the minimum of $n$ independent and identically distributed random variables having the common distribution $F$, i.e., $Y \sim \min \left\{C_{1}, \ldots, C_{n}\right\}$. It follows that $Y$ has the cumulative distribution function $G(c)=1-(1-F(c))^{n}$ and the probability density function $g(c)=n \cdot(1-F(c))^{n-1} f(c)$. Also, let $\bar{G}(c)=1-G(c)$. For simplicity of exposition, we assume that $f(c)>0$ for all $c \in[\underline{c}, \bar{c}]$. Lastly, we denote an indicator function by $I\{\cdot\}$.

The value of the product to the buyer is denoted by $v$. We assume that $v$ is public information, thus known to every seller, and lies above the support of $F$, i.e., $\bar{c} \leq v<\infty$. (We can easily extend our model to the scenario where the value of the product is uncertain to both the buyer and sellers, in which case, we use $v$ to denote the expected value of the 
product for the risk-free buyer.) The assumption of publicly available $v$ is applicable if the suppliers have long-term relationships with the buyer, if suppliers can infer the value of the product from market conditions, or if the value is equally uncertain to both the buyer and the suppliers.

We take the viewpoint of the buyer, who wants to maximize her expected profit. Maximizing the buyer's expected profit is an objective commonly employed in the optimal mechanism design literature. In this literature, a procurement mechanism refers to the determination of allocation and payment, where the outcome depends only on the bids submitted by the sellers. In this paper, however, we do not restrict our attention to the class of procurement mechanisms; instead, we allow that the outcome may also depend on the sellers' costs, possibly by auditing private information. We use the term procurement system to refer to this broader class of procurement methods. Examples of procurement systems include auctions and bargaining (discussed in Section 3).

In this paper, we study a procurement system that combines auction and bargaining sequentially. In our model, the first-phase auction is used to select the winning seller, and the second-phase bargaining determines the price (payment), which depends on both the bids submitted during the auction phase and the winning seller's cost. We call this an auctionbargaining (A-B) procurement system. In the first phase, each seller $i$ observes his ex post $\operatorname{cost} C_{i}=c$, which is private information known to him only, and then submits a sealed bid to the buyer. This bid represents a price at which the seller is willing to sell the product, should the buyer find it agreeable. The buyer selects the seller with the most competitive bid (i.e., the lowest bid price). Once the winning seller is chosen, the buyer can purchase the product at that price, or enter into second-phase bilateral bargaining with the winning seller for a possibly better price. As soon as the winning seller is determined at the end of the first phase, and prior to entering the bargaining phase, we assume that the buyer discovers the true cost to the winning bidder either through additional investigation or auditing the cost structure of the seller. (The notion of a post-auction audit has been used in multi-attribute auctions; see the scoring rule auction by Che [11and 12] and its application in Beil and Wein [2], Cachon and Zhang [9], Benjaafar et al. [3], and Wan and Beil [42].) We also assume that the buyer obtains this information without additional effort; we can easily extend our model to the case of positive cost associated with this additional effort by comparing it to the benefit of the bargaining phase. (Without the post-auction audit, the analysis of the model becomes quite involved as in Wang [43], and it does not yield results that are as simple as 
those presented in this paper.) At any time during the bargaining process, the buyer can purchase the product from the seller at the winning bid, which acts as an "outside option" that the buyer can exercise. Thus, the final price is bounded below by the winning seller's bid and above by the value $v$ of the product to the buyer. Since the auction phase of the A-B model is conducted using a first price sealed-bid format, we refer to this model as the first price sealed-bid A-B model, or simply the first price A-B model.

If the first-phase auction is instead conducted using an open descending-price auction, then we refer to this model as the the second price open-bid A-B model, or the second price A-B model. Here, the buyer starts the bidding process at a high price, and continuously lowers the price. Each bidder is initially active in the bidding process, and continues to remain active as long as he is willing to sell the product to the seller at the current bid price, and drops out when the current bid is no longer attractive. The bidding stops when there remains exactly one seller, who becomes the winning bidder. The buyer enters a bargaining process with the winning bidder, and the winning bid acts as an outside option for the buyer, as in the first price A-B model.

We remark that our intention is not to design the optimal procurement system, departing from the main focus of the mechanism design literature of economics. (For the buyer's profitmaximizing mechanism in the presence of the post-auction audit, it is possible to design a mechanism where the audit eliminates information asymmetry and the buyer extracts full rent; see Eső and Szentes [19]. However, such a mechanism is relatively complicated and may not be suited for business practice.) Rather, we restrict our attention to the sequential auction-bargaining system, which is simple and easy to implement in practice.

\section{Preliminaries}

Both auction and bargaining have been extensively studied in the literature. In this section, we summarize some of the results in the auction theory (Section 3.1) and the bargaining theory (Section 3.2), and establish elementary properties. These results will be useful later in analyzing the auction-bargaining (A-B) system, and also in comparing this system to the auction-only or bargaining-only systems. 


\subsection{Auction}

In a single-unit procurement auction, also known as a reverse auction, many potential sellers bid for the right to procure to a single buyer. The seller with the lowest bid wins the auction, and the payment to the seller is set by the lowest bid (in the first price auction), or by the second lowest bid (in the second price auction). Under the assumption that the sellers' opportunity costs are independent and identically distributed, the auction has been well analyzed in the literature (see, for example, a textbook by Krishna [26]). We review some classical results here.

We consider the symmetric bidding strategy, denoted by $\beta(c)$, where $c$ is the ex post opportunity cost of a seller. Let $\pi_{A}$ and $\Pi_{A}$ be the expected payment and expected profit made by the buyer. Recall that $C_{i}$ 's are independent and identically distributed, and $\bar{G}$ denotes the complementary cumulative distribution function of $Y \sim \min \left\{C_{1}, \ldots, C_{n}\right\}$.

Lemma 3.1. In the first price or the second price procurement auction without the buyer's reserve price,

$$
\begin{aligned}
\beta(c) & = \begin{cases}\mathbb{E}[Y \mid Y>c] & \text { in the first price auction } \\
c, & \text { in the second price auction }\end{cases} \\
\pi_{A} & =(n+1) \cdot \mathbb{E}\left[\mathbb{E}\left[Y \cdot I\left\{Y>C_{n+1}\right\} \mid C_{n+1}\right]\right], \\
\Pi_{A} & =v-\pi_{A} .
\end{aligned}
$$

See, for example, Section 2.3 of Krishna [26] for the proof of Lemma 3.1. Also, notice that as long as $f(c)>0$ for $c \in[\underline{c}, \bar{c}], \beta(c)$ is a strictly increasing function of $c$. In the above lemma, the expected payment $\pi_{A}$ made by the buyer is the same for both the first price and the second price auctions, and this result is a consequence of the celebrated Revenue Equivalence Theorem.

In the auction theory literature, the analysis and optimization of the reserve price has been well-studied. Suppose that at the beginning of the auction, the buyer announces a reserve price $r$, above which she commits herself not to pay. Let $\beta^{r}$ be the symmetric equilibrium bidding strategy under the reserve price $r$ in the auction only model. If $r<\bar{c}$, the analysis uses $\beta^{r}(r)=r$ as a boundary condition instead of $\beta(\bar{c})=\bar{c}$. Let $r_{A}^{*}$ denote the optimal reserve price that maximizes the buyer's expected profit in the auction only model. Let $\pi_{A}^{r}$ and $\Pi_{A}^{r}$ denote the expected payment and profit of the buyer, respectively. Note that the buyer can procure the product only if there exists at least one seller who bids below the reserve price $r$, which occurs with probability $1-(1-F(r))^{n+1}$. 
Lemma 3.2. In the first price or the second price procurement auction with the buyer's reserve price $r$,

$$
\begin{aligned}
\beta^{r}(c) & = \begin{cases}\mathbb{E}[\min \{Y, r\} \mid Y>c] & \text { in the first price auction with } \underline{c} \leq c \leq r \\
c, & \text { in the second price auction with } \underline{c} \leq c \leq r \\
\infty, & \text { if } c>r\end{cases} \\
\pi_{A}^{r} & =(n+1) \cdot \mathbb{E}\left[\mathbb{E}\left[\min \{Y, r\} \cdot I\left\{Y>C_{n+1}\right\} \mid C_{n+1}\right] \cdot I\left\{C_{n+1} \leq r\right\}\right], \\
\Pi_{A}^{r} & =\left[1-(1-F(r))^{n+1}\right] \cdot v-\pi_{A}^{r} .
\end{aligned}
$$

Furthermore, the optimal reserve price $r=r_{A}^{*}$ satisfies

$$
v-r=\frac{F(r)}{f(r)} .
$$

See, for example, Section 2.5 of Krishna [26] for the proof of Lemma 3.2. Also, notice that if $F(r) / f(r)$ is weakly increasing in $r$, the solution to the above equation is unique.

\subsection{Bargaining}

We review Rubinstein [36]'s bilateral bargaining model of the alternating offers under complete information. Consider a bargaining game between the buyer with the valuation $v$ and the seller with the opportunity $\operatorname{cost} c$, where $v \geq c$. Let $1-\lambda$ be the bargaining power of the seller, where $\lambda \in[0,1]$. (The bargaining power depends on the relative discount rates of the buyer and the seller, and also on which player first proposes a take-it-or-leave-it offer. If the seller proposes first, and $\delta_{s}, \delta_{b} \in(0,1)$ denote the discount factors of the seller and the buyer, then $1-\lambda=\left(1-\delta_{b}\right) /\left(1-\delta_{s} \delta_{b}\right)$. Note that the seller's bargaining power $1-\lambda$ is increasing in $\delta_{s}$ and decreasing in $\delta_{b}$.) We suppose that $v, c$ and $\lambda$ are public information.

Lemma 3.3. In the unique subgame perfect equilibrium of the alternating-offers bargaining game, the pricing outcome function is given by

$$
\gamma(c)=\lambda c+(1-\lambda) v
$$

Thus, if the seller has as much bargaining power as possible, i.e., $1-\lambda=1$, the price outcome of bargaining is $v$, capturing all the gains from the trade and leaving zero profit to the buyer. The proof of Lemma 3.3 is standard and can be found, for example, in Rubinstein [36] and Osborne and Rubinstein [35]. Note that the seller makes profit of $(1-\lambda)(v-c)$, which is bargaining power times the gains from the trade, whereas the buyer earns $\lambda(v-c)$. Lemma 3.3 implies that a strong bargaining power of the seller (with a low value of $\lambda$ ) results in a 
high negotiated price $\gamma(c)$. While the price depends on both $c$ and $v$, we hereafter suppress its dependency on $v$ for the simplicity of notation by treating the buyer's valuation $v$ as fixed.

We now consider the case with multiple sellers, in which the buyer bargains with the sellers in a sequential manner. As before, the buyer's valuation $v$ is public information. We also suppose that the opportunity costs and bargaining powers of the sellers $\left(c_{i}\right.$ and $\left.1-\lambda_{i}\right)$ are also public. (This is an additional assumption to those assumptions given in Section 3.1.)

At any point, the buyer may continue bargaining with the current seller, or discontinues current bargaining and start bargaining with a new seller. Once the buyer aborts bargaining with a seller, she cannot re-enter another round of bargaining with the same seller at a later point in time. For our discussion in this section, suppose that the sequence of sellers for bargaining is exogenously fixed, and the objective is to minimize the buyer's payment (the cost of bargaining is negligible).

To analyze this sequential bargaining game, we first consider a simpler case with two sellers. Let $\gamma_{i}(c)=\lambda_{i} \cdot c+\left(1-\lambda_{i}\right) \cdot v$, where $1-\lambda_{i}$ represents the bargaining power of seller $i$. If the buyer bargains with the second seller (seller 2) only, then the outcome of the price is $\gamma_{2}\left(c_{2}\right)$. Thus, in bargaining with the first seller (seller 1), the buyer has an option of aborting bargaining with seller 1 and then starting bargaining with seller 2. This option is analytically equivalent to exercising an option to purchase from buyer 2 at price $\gamma\left(c_{2}\right)$. During the bargaining with the first seller, this option involves procuring the product from someone not involved in the current bargaining process, and it is referred to as an outside option in the bargaining theory literature. (By contrast, the outside option refers to an option to purchase from the seller currently being negotiated. See Binmore [4], Shaked [38] and Muthoo [31] for details.) If $\gamma_{1}\left(c_{1}\right)<\gamma_{2}\left(c_{2}\right)$, then the buyer will bargain with seller 1 to reach the negotiated price of $\gamma_{1}\left(c_{1}\right)$; if $\gamma_{1}\left(c_{1}\right)>\gamma_{2}\left(c_{2}\right)$, then either the first seller will propose the price of $\gamma_{2}\left(c_{2}\right)$, or the buyer will immediately move to seller 2 for bargaining. Thus, the price that the buyer pays is $\min \left\{\gamma_{1}\left(c_{1}\right), \gamma_{2}\left(c_{2}\right)\right\}$. Extending the analysis to $n+1$ sellers (where $n \geq 1$ ), we can easily observe that the pricing outcome function of the sequential bargaining game is $\min _{i} \gamma_{i}\left(c_{i}\right)$. Note that the expression for the price is independent of the sequence of sellers. It is shown in a similar framework that the buyer is indifferent to the sequence of bargaining while the sellers prefer to bargain earlier (Nagarajan and Bassok [33], Nagarajan and Sosic [34]). 
Let $\pi_{B}$ be the expected payment of the buyer, by taking the expected value with respect to all possible realizations of $\left(C_{1}, \ldots, C_{n}, C_{n+1}\right)$, and let $\Pi_{B}$ be the expected profit of the buyer.

Lemma 3.4. In the sequential bargaining model,

$$
\begin{aligned}
\pi_{B} & =\sum_{i=1}^{n+1} \mathbb{E}\left[\mathbb{E}\left[\gamma_{i}\left(C_{i}\right) \cdot \mathbb{P}\left[\gamma_{i}\left(C_{i}\right) \leq \gamma_{j}\left(C_{j}\right), \forall j \neq i\right] \mid C_{i}\right]\right], \\
\Pi_{B} & =v-\pi_{B} .
\end{aligned}
$$

Furthermore, if $\lambda_{i}=\lambda$ for each $i$, then $\min _{i}\left\{\gamma_{i}\left(c_{i}\right)\right\}=\gamma\left(\min _{i} c_{i}\right)$, and it follows that

$$
\pi_{B}=(n+1) \cdot \mathbb{E}\left[\gamma\left(C_{n+1}\right) \cdot \bar{G}\left(C_{n+1}\right)\right]
$$

Both in the auction model and in the sequential bargaining model, the buyer procures the product from one of the competing sellers, but the expected payments are not identical. The celebrated revenue equivalence result is not applicable here since, in the bargaining model, the payment by the buyer to the winning seller depends on the bargaining powers of all the sellers. Thus, the bargaining model is not a procurement mechanism in the classical sense. In fact, we caution the reader that these two models should not be compared directly since the auction-only model assumes that the buyer does not know the seller's cost, contrary to the assumption made in the sequential bargaining model.

\section{Analysis of the Auction-Bargaining (A-B) Model}

In this section, we analyze the auction-bargaining (A-B) model described in Section 2. We first consider the first price A-B model where the sellers have identical bargaining power $1-\lambda$ and the buyer does not set any reserve price (Section 4.1). We find a symmetric bidding strategy equilibrium, and derive an expression for the expected payment by the seller, which we compare to the auction-only and sequential bargaining-only counterparts. We also consider the model where a reserve price is set by the buyer in the auction phase (Section 4.2), and the second price A-B model (Section 4.3).

\subsection{First Price A-B Model}

The A-B model consists of two phases: the auction phase followed by the bargaining phase. The bids submitted by sellers during the auction phase are not only used to determine the 
winning seller (with the lowest bid), but also acts as a price for the outside option which the buyer can exercise subsequently in the bargaining phase. Thus, when a seller submits his bid, he strikes a balance between increasing the probability of winning in the auction phase and decreasing the price of the buyer's option in the event that he becomes the winning bidder. In this section, we study the symmetric bidding strategy of sellers in the auction phase of the first price A-B model.

We suppose that the sellers have identical bargaining power, i.e., $1-\lambda_{i}=1-\lambda$ for all $i$. We are interested in the symmetric bidding strategy of the first phase, which we denote by $\psi$. It is straightforward to show that any reasonable bidding strategy satisfies $\psi(c) \geq c$; otherwise, the bidder's profit would be negative. We assume that $\psi$ is a strictly increasing function of the bidder's opportunity $\operatorname{cost} c$. Recall that $\beta$ and $\gamma$ represent the symmetric bidding strategy in the first price auction-only model and the pricing outcome function of the bilateral bargaining game, respectively. We assume a technical condition that these two functions $\beta$ and $\gamma$ intersect finitely many times in $[\underline{c}, \bar{c}]$.

We start with the following lemma stating the relationship of the $\psi$ function and $\gamma$ function. The proof of this result is based on the following observation that if a bidder with cost $c$ places an auction phase bid larger than $\gamma(c)$, then the price outcome in the subsequent bargaining phase cannot exceed $\gamma(c)$. Thus, by decreasing his bid price to $\gamma(c)$, the bidder increases the probability of his winning without affecting his profit in the case that he wins the auction phase. The proof of Lemma 4.1 and all other proofs are located in the e-companion to this paper.

Lemma 4.1. In the first price $A-B$ model, any strictly increasing equilibrium bidding strategy $\psi$ in the auction phase satisfies, for all $c \in[\underline{c}, \bar{c}]$,

$$
\psi(c) \leq \gamma(c)
$$

If the bidder with ex post cost $c$ wins the auction phase, then the price outcome of the subsequent bargaining phase is the minimum of his winning bid in the first phase and $\gamma(c)$. Thus, as a corollary of the above lemma, the winning bidder receives $\psi(c)$ from the buyer.

In Theorem 4.2 below, we present a symmetric equilibrium strategy $\psi$ in the auction phase. It turns out that this equilibrium strategy is given by a simple expression involving $\beta$ and $\gamma$. However, we introduce a technical condition that is required by Theorem 4.2. Indeed, we show later that if this condition fails to satisfy, then $\psi$ may not exist. Recall 
that $1-\lambda$ is the bargaining power of the seller, and $\lambda$ is the slope in the definition of the bargaining outcome price function $\gamma$. Also, recall that $g$ and $G$ represent the probability density function and cumulative distribution function of $Y \sim \min \left\{C_{1}, \ldots, C_{n}\right\}$, respectively. For any given bidding strategy $\psi:[\underline{c}, \bar{c}] \rightarrow \Re^{+}$, define $\Gamma^{\psi}$ to be a subset of $[\underline{c}, \bar{c}]$ where the inequality in Lemma 4.1 is tight, i.e., $\Gamma^{\psi}=\{c \in[\underline{c}, \bar{c}] \mid \psi(c)=\gamma(c)\}$.

Condition A. A first phase bidding strategy $\psi:[\underline{c}, \bar{c}] \rightarrow \Re^{+}$satisfies the following condition: for any interior point c of $\Gamma^{\psi}$,

$$
\lambda \geq \frac{g(c)}{\bar{G}(c)}(\gamma(c)-c)
$$

Condition $\mathrm{A}$ is a technical assumption required in proving Theorem 4.2, which provides a simple expression for the first phase equilibrium bidding strategy. A sufficient condition for this bidding strategy to satisfy Condition A will be given later in Lemma 4.3. In the uniform $[0,1]$ distribution case, this sufficient condition is equivalent to the fact that the seller's bargaining power $(1-\lambda)$ does not exceed $1 /(n+1)$. This is not unreasonable since there are a total of $n+2$ agents in the system $(n+1$ sellers and one buyer).

Theorem 4.2. Let $\psi$ be a strictly increasing function defined on $[\underline{c}, \bar{c}]$ by

$$
\psi(c)=\min \{\beta(c), \gamma(c)\}
$$

If $\psi$ satisfies Condition $A$, then it is a symmetric equilibrium bidding strategy in the first phase of the first price $A-B$ model. Furthermore, if $\psi$ does not satisfy Condition $A$, then it is not a symmetric bidding strategy.

Theorem 4.2 implies that a seller's bid is more aggressive in the A-B model than in the auction-only model. The upper bound of $\gamma$ on $\psi$ (Lemma 4.1) partly explains the aggressiveness in the sellers' first-stage bidding strategy. Each bidder does not submit any bid higher than the corresponding $\gamma$ value, and furthermore he knows that the other bidders will be more aggressive than the auction-only model. It is also interesting to note that as the sellers' bargaining power $1-\lambda$ decreases, $\gamma(c)$ decreases and sellers bid more aggressively. Thus, the degree of the sellers' aggressiveness in the first phase represents the relative weakness in their bargaining position against the buyer. In an extreme case where $1-\lambda$ is $0, \gamma(c)$ becomes $c$, i.e., each seller bids his true cost and gains zero profit. In the other extreme case of $1-\lambda=1$, it follows $\gamma(c)=v$ and $\psi(c)$ becomes $\beta(c)$, a symmetric equilibrium bidding strategy of the auction-only model. 
The following result provides a sufficient condition for Condition A, which is easy to verify when $\beta^{\prime}(c)$ is readily available.

Lemma 4.3. A first-phase bidding strategy $\psi=\min \{\beta(c), \gamma(c)\}$ satisfies Condition $A$ if $\lambda \geq \beta^{\prime}(c)$ for all $c \in[\underline{c}, \bar{c}]$.

Lemma 4.3 is useful when the bidding strategy $\beta$ of the auction-only model is absolutely continuous. For example, if $F$ is a uniform distribution, then $\beta$ is linear and one can easily verify whether $\lambda \geq \beta^{\prime}(c)$ is satisfied. (We remark that Lemma 4.3 also holds if the condition $\gamma^{\prime}(c)=\lambda \geq \beta^{\prime}(c)$ holds only for interior points of $\Gamma^{\psi}$; however this condition is stated such that it is independent of $\psi$.)

The following corollary compares the expected payment of the buyer in the first price A-B model under the symmetric equilibrium given in Theorem 4.2 to the expected payment in the auction-only model or in the sequential bargaining model. It states that the A-B model generates higher profit to the buyer than the auction-only or sequential bargaining model. It can be explained by the fact that each seller's bid in the A-B model (see Theorem 4.2 ) is less than or equal to both his corresponding bid in the auction-only model or the price outcome in the bargaining model. For a given symmetric equilibrium bidding strategy $\psi$ for the auction phase, let $\pi_{A B}^{\psi}$ and $\Pi_{A B}^{\psi}$ denote the expected payment and profit of the buyer, respectively. (Recall that the pair of $\pi_{A}$ and $\Pi_{A}$ and the pair of $\pi_{B}$ and $\Pi_{B}$ have been similarly defined for the auction-only model and the sequential bargaining-only model, respectively. Also, recall that $C_{n+1}$ has a cumulative density function $F$.)

Corollary 4.4. In the first price $A-B$ model, suppose that $\psi(c)=\min \{\beta(c), \gamma(c)\}$ is a strictly increasing symmetric equilibrium satisfying Condition A. Then, the buyer's expected payment and profit are given by

$$
\begin{aligned}
\pi_{A B}^{\psi} & =(n+1) \cdot \mathbb{E}\left[\min \left\{\beta\left(C_{n+1}\right), \gamma\left(C_{n+1}\right)\right\} \cdot \bar{G}\left(C_{n+1}\right)\right] \\
\Pi_{A B}^{\psi} & =v-\pi_{A B}^{\psi} .
\end{aligned}
$$

Furthermore, $\Pi_{A B}^{\psi} \geq \max \left\{\Pi_{A}, \Pi_{B}\right\}$.

The proof of Corollary 4.4 is straightforward and thereby omitted. To establish the inequality of $\Pi_{A B}^{\psi} \geq \max \left\{\Pi_{A}, \Pi_{B}\right\}$, we observe from Lemmas 3.1 and 3.4 that $\pi_{A}=(n+$ $1) \cdot \mathbb{E}\left[\beta\left(C_{n+1}\right) \cdot \bar{G}\left(C_{n+1}\right)\right]$ and $\pi_{B}=(n+1) \cdot \mathbb{E}\left[\gamma\left(C_{n+1}\right) \cdot \bar{G}\left(C_{n+1}\right)\right]$. 
We recall from Section 3 that the ranking between the auction-only model and the sequential bargaining model depends on the model parameters. Corollary 4.4 above establishes that, for the risk-neutral buyer, the equilibrium of the A-B model given in Theorem 4.2 is preferable to both the auction-only model and the sequential bargaining model. Thus, if this equilibrium is the unique equilibrium, then it is not necessary for the buyer to study model parameters when she is faced with the question of which procurement system would result in the largest expected profit. The A-B model results in the buyer's expected profit that is higher than the expected profit of the other two models studied in Section 3. We caution the reader that this result is based on our modeling assumption that running an auction or a bargaining round is costless. When the cost of running a bargaining round is not negligible, we determine whether the first price A-B model is still preferable to the auction-only model by comparing this cost to the difference in expected profits, $\Pi_{A B}^{\psi}-\Pi_{A}$. Similarly, when the cost of running an auction is not negligible, we compare the first price A-B model to the sequential bargaining model by considering $\Pi_{A B}^{\psi}-\Pi_{B}$. We also note that while the first price A-B model runs only one bargaining round, the sequential bargaining model can possibly run multiple rounds of bargaining if the buyer does not discover a seller's cost until she enters bargaining with the seller. Thus, the first price A-B model has an advantage of identifying the most competitive seller, with whom the buyer can bargain. (See Section 5 for details.)

While Theorem 4.2 shows one equilibrium bidding strategy for the A-B model, we now consider the possibility of other symmetric increasing bidding strategies for $\psi$. Before we continue with our analysis, we re-visit the symmetric equilibrium bidding strategy in the first price auction-only model, discussed in Section 3.1. For this model, $\beta(c)=\mathbb{E}[Y \mid Y>c]$ is an equilibrium bidding strategy, and it is a solution to the following differential equation:

$$
0=\left.\frac{\partial}{\partial z}[\bar{G}(z) \cdot(\beta(z)-c)]\right|_{z=c}=-g(c)(\beta(c)-c)+\bar{G}(c) \frac{\partial}{\partial c} \beta(c) .
$$

Solving this differential equation, we obtain a family of non-intersecting solutions characterized by the constant of integration $K \in(-\infty, \infty)$ :

$$
\beta_{K}(c)=\mathbb{E}[Y \mid Y>c]+\frac{K}{\bar{G}(c)} .
$$

If $K=0$, then $\beta_{K}$ corresponds to $\beta$ given above in Lemma 3.1. If $K>0$, then it can be shown that $\beta_{K}$ forms a symmetric equilibrium for the first price auction-only model, but it has the undesirable property that it approaches $\infty$ as $c$ approaches $\bar{c}$ from the left. If 
$K<0$, then $\beta_{K}$ is not monotone in $[\underline{c}, \bar{c}]$; however, it is used to define the first price bidding strategy when a buyer specifies a reserve price. (When the reserve price is $r \in[\underline{c}, \bar{c}]$, then the equilibrium bidding strategy is given by $\beta^{r}=\beta_{K}$, where $\beta_{K}(r)=r$.) The above definition of $\beta_{K}$ proves to be convenient in defining bidding strategies for the first price A-B model.

Theorem 4.5. In the first price A-B model, suppose that a continuous and increasing function $\psi$ satisfies Condition $A$. Then, $\psi$ is an equilibrium bidding strategy for the auction phase of the first price $A-B$ model if and only if

(i) $\psi(c) \leq \gamma(c)$ for $c \in[\underline{c}, \bar{c}]$, and

(ii) there exists $\left\{a_{0}, a_{1}, \ldots, a_{m}\right\}$ satisfying $\underline{c}=a_{0} \leq a_{1} \leq a_{2} \leq \cdots \leq a_{m}=\bar{c}$ such that for each interval $I_{i}=\left[a_{i-1}, a_{i}\right]$ where $i=1, \ldots, m$, either

$$
\psi(c)=\beta_{K_{i}}(c) \text { for some } K_{i} \in(-\infty, \infty), \quad \text { or } \quad \psi(c)=\gamma(c) .
$$

Furthermore, $\psi(\bar{c})=\bar{c}$.

Since the family of curves $\left\{\beta_{K} \mid K \in(-\infty, \infty)\right\}$ parameterized by $K$ are nonintersecting and $\psi$ is continuous, we can assume without loss of generality that the sequence of intervals $I_{i}$ 's alternatively satisfies $\psi(c)=\beta_{K_{i}}(c)$ or $\psi(c)=\gamma(c)$. Thus, Theorem 4.5 shows that an equilibrium bidding strategy $\psi$ in the first price A-B model consists by alternating $\gamma$ and $\beta_{K_{i}}$ functions, provided that Condition $\mathrm{A}$ is satisfied whenever $\psi$ coincides with $\gamma$. This result is useful in constructing $\psi$. The next result states that Condition A must be satisfied for every symmetric equilibrium bidding strategy.

Lemma 4.6. If a continuous increasing function $\psi$ does not satisfy Condition $A$, then $\psi$ is not a symmetric equilibrium bidding strategy in the first phase of the A-B model.

We now remark on statement (ii) in Theorem 4.5, focusing on the right-most interval $I_{m}=\left[a_{m-1}, a_{m}\right]$. For $c \in I_{m}$, either $\psi(c)=\beta_{K_{m}}(c)$ or $\psi(c)=\gamma(c)$. In the former case, we must have $K_{m}=0$. (Otherwise, strictly positive $K_{m}$ implies $\lim _{c \uparrow \bar{c}} \beta_{K_{m}}(c) \rightarrow \infty$, contradicting the definition of $\psi$ in Theorem 4.2, and strictly negative $K_{m}$ implies decreasing $\beta_{K_{m}}$, contradicting the monotonicity of $\psi$.) In the latter case, we have $\psi(\bar{c})=\gamma(\bar{c})$, which is possible only if $\psi(\bar{c})=\gamma(\bar{c})=\beta_{0}(\bar{c})=\bar{c}$. Thus, we obtain $\psi(c) \leq \bar{c}$, which states that the first-phase bid would not be higher than the highest possible cost, which is a reasonable outcome. 
The following result is useful in constructing another symmetric equilibrium bidding strategy from a given equilibrium. In the first proof of Corollary 4.7, we replace a sequence of a symmetric bidding strategy $\psi$ with $\gamma$; in the second part, we replace it with $\beta_{K}$ for some $K$. The proof of Corollary 4.7 follows from Theorem 4.5 and Lemma 4.6, and is omitted.

Corollary 4.7. Let $\psi$ be an equilibrium symmetric bidding strategy for the auction phase of the first price $A-B$ model. Let $s$ and $t$ satisfy $\underline{c} \leq s<t \leq \bar{c}$, and let $\psi^{\circ}$ be an increasing function such that $\psi^{\circ}(c)=\psi(c)$ for $c \notin(s, t)$.

(a) Suppose both $\psi(s)=\gamma(s)$ and $\psi(t)=\gamma(t)$ hold, and inequality (1) holds for $c \in(s, t)$. If $\psi^{\circ}(c)=\gamma(c)$ for $c \in(s, t)$, then $\psi^{\circ}$ is an equilibrium bidding strategy with $\Pi_{A B}^{\psi^{\circ}} \leq \Pi_{A B}^{\psi}$.

(b) Suppose both $\psi(s)=\beta_{K}(s)$ and $\psi(t)=\beta_{K}(t)$ hold for some $K$, and $\psi(c) \leq \beta_{K}(c)$ holds for $c \in(s, t)$. If $\psi^{\circ}(c)=\beta_{K}(c)$ for $c \in(s, t)$, then $\psi^{\circ}$ is an equilibrium bidding strategy with $\Pi_{A B}^{\psi^{\circ}} \leq \Pi_{A B}^{\psi}$.

Corollary 4.7 shows that there exists a partially ordered ranking among symmetric equilibria with respect to the expected profit of the buyer. Corollary 4.8 below shows that there is a biggest and smallest element in this partially ordered set, through yet another method of constructing an equilibrium.

Corollary 4.8. Let $\psi^{1}$ and $\psi^{2}$ be equilibrium symmetric bidding strategies for the auction phase of the first price $A-B$ model. Then, both $\psi^{m}(c)=\min \left\{\psi^{1}(c), \psi^{2}(c)\right\}$ and $\psi^{M}(c)=$ $\max \left\{\psi^{1}(c), \psi^{2}(c)\right\}$ are also equilibrium symmetric bidding strategies. Furthermore,

$$
\Pi^{\psi^{M}} \leq \min \left\{\Pi^{\psi^{1}}, \Pi^{\psi^{2}}\right\} \leq \max \left\{\Pi^{\psi^{1}}, \Pi^{\psi^{2}}\right\} \leq \Pi^{\psi^{m}} .
$$

The proof of Corollary 4.8 is straightforward and thereby omitted.

Notice that Theorem 4.5 implies the possibility of multiple equilibrium bidding strategies, each characterized by a collection of intervals and $K_{i}$ values. The profit-comparison result of Corollary 4.4 (i.e., $\Pi_{A B}^{\psi} \geq \max \left\{\Pi_{A}, \Pi_{B}\right\}$ ) corresponds to a particular equilibrium (given by $\psi(c)=\min \{\beta(c), \gamma(c)\}$ ), and this result may not hold with other equilibrium bidding strategies. The following theorem shows that the above comparison of profit holds for any symmetric equilibrium bidding strategy.

Theorem 4.9. For any increasing and continuous symmetric equilibrium bidding strategy $\psi$ in the $A$-B model that satisfies Condition $A$, we have $\Pi_{A B}^{\psi} \geq \max \left\{\Pi_{A}, \Pi_{B}\right\}$. 
Example: Uniform $[0,1]$ Cost in the First Price A-B Model.

We consider an example of uniform opportunity costs, where each $C_{i}$ has a uniform distribution on $[0,1]$, with $v \geq 1$. In this uniform [0,1] cost case, it turns out that Condition A is satisfied if and only if $\lambda \geq n /(n+1)$, and in this case, we obtain the following uniqueness result.

Theorem 4.10. In the first price $A-B$ model, suppose that sellers' opportunity costs have uniform $[0,1]$ distribution and $\lambda>n /(n+1)$. Then, there exists a unique continuous strictly increasing symmetric equilibrium bidding strategy for the auction phase, given by

$$
\psi(c)=\min \left\{\beta_{0}(c), \gamma(c)\right\} .
$$

The proof of Theorem 4.10 is based on the observation that whenever $\gamma(c)<\beta_{0}(c)$, any $\beta_{K}$ intersecting with $\gamma$ at $c$ has the property $\beta_{K}^{\prime}(c)<\lambda=\gamma^{\prime}(c)$.

We now describe in detail the symmetric equilibrium bidding strategy given in Theorem 4.10. We compute the buyer's expected profit in the A-B model, and compare it to the auction-only model and the sequential bargaining model. Since each seller's cost is uniformly distributed, we obtain $\bar{G}(c)=(1-c)^{n}$. Thus, the auction phase bidding strategy is given by $\psi(c)=\min \left\{\beta_{0}(c), \gamma(c)\right\}$ where

$$
\beta_{0}(c)=\frac{1}{n+1}+\frac{n}{n+1} \cdot c \quad \text { and } \quad \gamma(c)=(1-\lambda) \cdot v+\lambda \cdot c .
$$

Since both $\beta_{0}$ and $\gamma$ are linear functions, they intersect at most once depending on the buyer's value $v$ and the seller's bargaining power $1-\lambda$. Let $s=\inf \{c \in[0,1] \mid \beta(c) \leq \gamma(c)\}$. (Since $n /(n+1)$ and $\lambda$ correspond to the slopes of $\beta_{0}$ and $\gamma, s$ represents the intersection of these two lines if $s \in(0,1)$.) Thus,

$$
\psi(c)=\min \{\beta(c), \gamma(c)\}= \begin{cases}\gamma(c), & \text { if } c \in[0, s) \text { or } c=s \in(0,1] \\ \beta(c), & \text { if } c \in(s, 1] \text { or } c=s \in[0,1) .\end{cases}
$$

Also, from Lemma 4.3 and $\lambda>n /(n+1)=\beta^{\prime}(c)$, it is easy to verify that $\psi$ satisfies Condition A.

We comment on the assumption $\lambda>n /(n+1)$. It is reasonable to expect that if there is only one potential seller, the bargaining powers of the seller and the buyer are similar, i.e., $\lambda \sim 1 / 2$. The seller's bargaining power typically decreases as the number of sellers increases. When there are $n+1$ potential sellers, there are a total of $n+2$ players in the 
market (including the buyer), and it is reasonable to expect that each seller's bargaining power satisfies $1-\lambda=1 /(n+2)$. In this case, $\lambda=(n+1) /(n+2)>n /(n+1)=\beta^{\prime}(c)$. Furthermore, if $\lambda>n /(n+1)$ does not hold, then by Lemma 4.6 there exists no strictly increasing equilibrium bidding strategy in the A-B model with the exception of $\psi(c)=\beta(c)$.



(a) $\lambda=0.75$, and $n=2$ (i.e., 3 sellers).

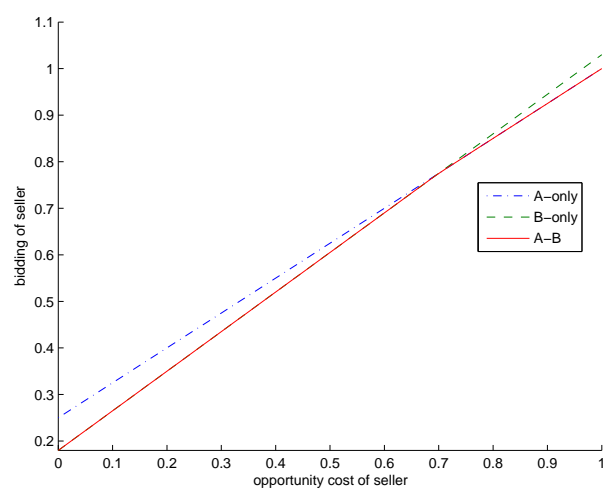

(b) $\lambda=0.85$, and $n=3$ (i.e., 4 sellers).

Figure 1: The symmetric equilibrium bidding strategy of the A-B Model (A-B) compared to the bidding strategy of the auction-only (A-only) model and the pricing outcome function of the sequential bargaining model (B-only). $v=1.2$.

Figure 1 shows the symmetric equilibrium bidding strategy of the A-B model in comparison to the symmetric equilibrium bidding strategy of the auction-only model as well as the pricing outcome function of the bargaining model. The expected profit $\Pi_{A B}^{\psi}$ of the buyer in the A-B model can be computed in a closed form. (See Appendix H for the detail.) Figure 2 shows that the expected profit of the buyer in the first price A-B model is higher than in the auction-only model or the sequential bargaining-only model. In addition, while it can be shown that the buyer's expected profit in the auction-only model or the sequential bargaining model can be shown to be concave with respect to the number of the sellers, it may fail to be concave in the A-B model.

It is interesting to observe from Figure 2 that it is not always more profitable for the buyer to add one more seller to the auction-only model than to have the second-phase of bargaining as in the A-B model. Consider, for example, the case where the number of sellers is 2 in Figure 2(b). This is in contrast to a conclusion of Bulow and Klemperer [8], in which the benefit of the second-phase ultimatum bargaining is always outweighed by the benefit of having an extra bidder. Our observation is consistent with a similar finding of Wang [43] for his auction-bargaining model, where he assumes a one-sided uncertainty of the private 
buyer's valuation in the bargaining phase. Whereas Bulow and Klemperer [8] do not consider the presence of an audit, both Wang [43] and our paper allow audit prior to the bargaining phase, thereby the buyer is equipped with more information to benefit from the auction phase. Thus, in these two papers, it is quite plausible that the benefit of bargaining may become preferable to attracting one more bidder.

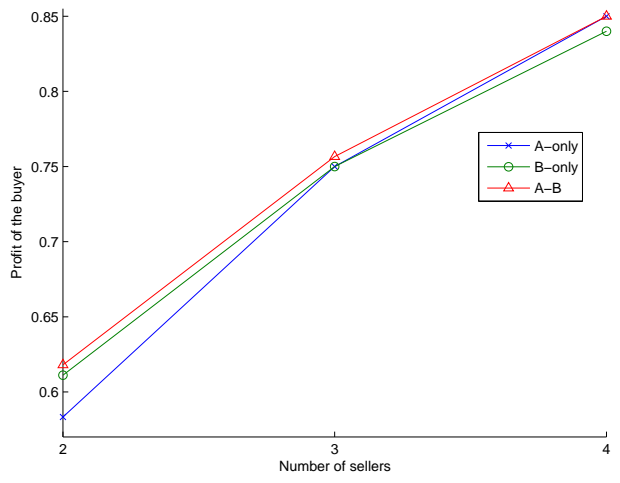

(a) $\lambda=(n+1) /(n+2)$ under $2,3,4$ sellers.

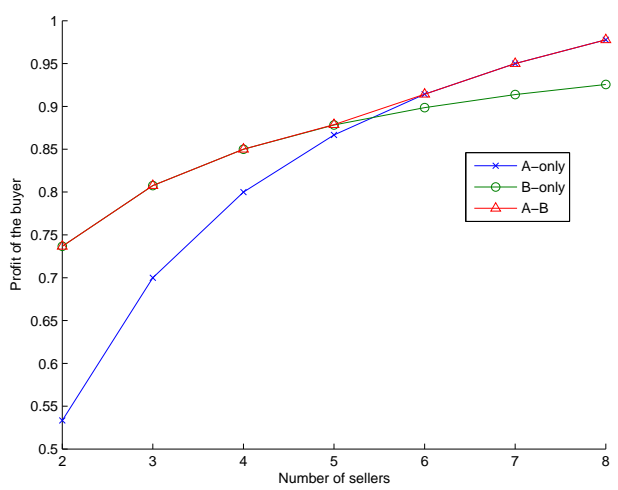

(b) $\lambda=0.85$ under $2, \ldots, 8$ sellers.

Figure 2: The expected profit of the buyer in the A-B model (A-B), compared to the auctiononly model (A-only) and the sequential bargaining model (B-only), as a function of the total number of sellers, $n+1 . v=1.2$.

\subsection{A-B Model with Reserve Price}

While we have assumed in Section 4.1 that the buyer does not set any reserve price, we now consider the case where the buyer announces a reserve price, over which she commits not to purchase the product from any of the sellers. This announcement is made before the bids are submitted in the first phase. By setting a reserve price, the buyer faces the risk of not being able to procure, but may increase her expected profit by encouraging sellers to bid more aggressively.

We use $r$ to denote the reserve set by the buyer. Since the buyer does not want to procure the product above its value $v$, we proceed by assuming $r \leq v$. Recall that $\beta^{r}$ represents the symmetric bidding strategy in the first price auction-only model with reserve price $r$. Also recall that $\gamma$ denotes the pricing outcome function of the bilateral bargaining game. We assume that these two functions $\beta^{r}$ and $\gamma$ intersect finitely many times in $[\underline{c}, r]$. Similar to the first price A-B model without reserve price, we introduce a technical condition that is required by Theorem 4.11 below. Recall that from the definition of $\Gamma, \Gamma^{\psi^{r}}=\left\{c \mid \psi^{r}(c)=\gamma(c)\right\}$. 
Condition B. A bidding strategy $\psi^{r}$ satisfies the following condition: for any c satisfying $\underline{c}<c<r$ in the interior point of $\Gamma^{\psi^{r}}$,

$$
\lambda \geq \frac{g(c)}{\bar{G}(c)}(\gamma(c)-c) .
$$

Note that Condition B is identical to Condition A except that the above inequality is required for $c \in(\underline{c}, r)$ only. The following theorem summarizes the main results for the first price A-B model with a reserve price.

Theorem 4.11. Let $\psi^{r}$ be a strictly increasing function defined on $[\underline{c}, r]$ by

$$
\psi^{r}(c)=\min \left\{\beta^{r}(c), \gamma(c)\right\} .
$$

If $\psi^{r}$ satisfies Condition B, then it is a symmetric equilibrium bidding strategy in the first phase of the first price $A-B$ model with reserve price $r$.

The proof of Theorem 4.11 is similar to the proof of Theorem 4.2, and is omitted. We observe from Theorem 4.11 that a seller's bid in the first phase of the A-B model is more aggressive than the auction-only model with same reserve price. This observation is analogous to the case without any reserve price (Section 4.1). Furthermore, note that the seller's bid $\psi^{r}(c)$ becomes more aggressive as $r$ decreases - just as in the auction-only model. We remark that Condition B is required to guarantee that $\psi^{r}$ forms an equilibrium.

The following corollary shows the expected profit of the buyer in the first price A-B model with reserve price. The result is immediate, and thus we omit the proof of the corollary.

Corollary 4.12. Under the conditions of Theorem 4.11, the buyer's expected payment and profit are given by

$$
\begin{aligned}
\pi_{A B}^{r} & =(n+1) \cdot \mathbb{E}\left[\bar{G}\left(C_{n+1}\right) \psi^{r}\left(C_{n+1}\right) \cdot I\left\{C_{n+1} \leq r\right\}\right], \\
\Pi_{A B}^{r} & =\left[1-(1-F(r))^{n+1}\right] v-\pi_{A B}^{r} .
\end{aligned}
$$

From Lemma 3.2 and Corollary 4.12, it is clear that, for the risk-neutral buyer, the A-B model with reserve price is preferable to the auction-only model with the same reserve price. We now consider the optimal reserve price which maximize the buyer's expected profit. Let $r_{A B}^{*}$ denote the optimal reserve price of the buyer in the first price A-B model. The first order condition for the optimal reserve price in the first price A-B model is derived in the following lemma. 
Lemma 4.13. Under the condition of Corollary 4.12, the optimal reserve price $r=r_{A B}^{*}$ satisfies the following equation:

$$
v-r=\frac{\int_{\underline{c}}^{r} I\left\{\beta^{r}(c) \leq \gamma(c)\right\} \cdot f(c) d c}{f(r)} .
$$

Note that the integral part of the above equation, $\int_{\underline{c}}^{r} I\left\{\beta^{r}(c) \leq \gamma(c)\right\} \cdot f(c) d c$, represents the probability that a seller's ex post cost $c$ satisfies both $\beta^{r}(c) \leq \gamma(c)$ and $c \leq r$. Lemma 4.13 states that the optimal reserve price in the auction-only model, $r_{A}^{*}$, is not necessarily the optimal reserve price in the A-B model. In fact, if $F(r) / f(r)$ is weakly increasing, then the unique optimal reserve price $r_{A}^{*}$ given in Lemma 3.2 can be shown to be a lower bound for $r_{A B}^{*}$. We obtain this result by comparing the equation in Lemma 3.2 to the equation in Lemma 4.13. In other words, in the A-B model, the buyer sets a less-aggressive reserve price than the auction-only model. We attribute this observation to the fact that the seller's bid is less important in the A-B model because the buyer learns the seller's cost before the second-phase bargaining process.

While Theorem 4.11 shows one equilibrium bidding strategy for the A-B model with reserve price, we now consider the possibility of other symmetric bidding strategies for $\psi^{r}$ as in the previous section. The following theorem is analogous to Theorem 4.5 and Lemma 4.6 , and we omit this proof.

Theorem 4.14. In the first price $A-B$ model with reserve price, suppose that a continuous increasing function $\psi^{r}$ satisfies Condition B. Then, $\psi^{r}$ is a symmetric equilibrium bidding strategy for the first phase if and only if

(i) $\psi^{r}(r)=r$

(ii) $\psi^{r}(c) \leq \gamma(c)$ for $c \in[\underline{c}, r]$

(iii) There exists $\left\{a_{0}, a_{1}, \ldots, a_{m}\right\}$ and $K_{i} \in(-\infty, \infty)$ for each $K_{i}$ such that $\underline{c}=a_{0} \leq a_{1} \leq$ $a_{2} \leq \cdots \leq a_{m}=r$ and for each $I_{i} \in\left[a_{i-1}, a_{i}\right]$, either

$$
\psi^{r}(c)=\beta_{K_{i}}(c) \text { for some } K_{i} \in(-\infty, \infty), \quad \text { or } \quad \psi^{r}(c)=\gamma(c)
$$

Furthermore, $\psi^{r}(c)=\min \left\{\beta^{r}(c), \gamma(c)\right\}$ for $c \in I_{m}$.

Also, if a continuous increasing function $\psi^{r}$ does not satisfy Condition $B$, then $\psi^{r}$ is not a symmetric equilibrium bidding strategy in the first phase of the $A$-B model with reserve price $r$. 
Example: Uniform [0,1] Cost in the A-B Model with Reserve Price.

We return to the example of the uniform $[0,1]$ opportunity cost. As before, we assume $\lambda>n /(n+1)$. We also suppose $\gamma(0) \leq \beta^{r}(0)$; otherwise, it can be shown that $\psi^{r}(c)=\beta^{r}(c)$, implying that the A-B model becomes the same as the auction-only model. The following lemma establishes that $\psi^{r}$ given in Theorem 4.11, $\psi^{r}(c)=\min \left\{\beta^{r}(c), \gamma(c)\right\}$, satisfies Condition $\mathrm{B}$, and is a unique first-phase bidding strategy for the first price A-B model with reserve price $r$.

Lemma 4.15. In the first price $A-B$ model with reserve price, suppose that the seller's opportunity costs are drawn from uniform $[0,1]$ distribution. Let $r \in[0,1]$ be the reserve price, and suppose that both $\gamma(0) \leq \beta^{r}(0)$, and $\lambda>n /(n+1)$ hold. Then, the symmetric equilibrium bidding strategy $\psi^{r}(c)$ for the first phase is unique and given by

$$
\psi^{r}(c)=\min \left\{\beta^{r}(c), \gamma(c)\right\}
$$

The proof of Lemma 4.15 is similar to Theorem 4.10, and is based on the observation that whenever $\gamma(c)<\beta^{r}(c)$, any $\beta_{K}$ intersecting with $\gamma$ at $c$ has the property $\beta_{K}^{\prime}(c)<\lambda=\gamma^{\prime}(c)$.

In the uniform $[0,1]$ cost case, it follows from Lemma 3.2 that

$$
\beta^{r}(c)=\frac{n c+1}{n+1}-\frac{(1-r)^{n+1}}{(n+1)(1-c)^{n}},
$$

and $\beta^{r}$ is concave with respect to $c$ in $[0, r]$. Since $\gamma(0) \leq \beta^{r}(0)$ and $\gamma(r) \geq r=\beta^{r}(r)$, there exists a unique intersection of $\beta^{r}$ and $\gamma$ in $[0, r]$. Let $s^{r} \in[0, r]$ such that $\beta^{r}\left(s^{r}\right)=\gamma\left(s^{r}\right)$. Figure 3 shows the optimal bidding strategy in the auction-only model with reserve price $r \in$ $\{0.5,0.8\}$ and the pricing outcome function of bargaining. We observe that the intersection of these two functions is unique for each value of $r$. From Lemma 4.15, $\psi^{r}(c)$ is given by the minimum of these two functions.

From Corollary 4.12, it is straightforward to verify that the expected payment from the buyer, $\pi_{A B}^{\psi^{r}}$, and the expected profit of the buyer, $\Pi_{A B}^{\psi^{r}}$, are

$$
\begin{aligned}
\pi_{A B}^{\psi^{r}} & =(n+1)\left(\int_{0}^{r}(1-c)^{n} \cdot \min \left\{\beta^{r}(c), \gamma(c)\right\} d c\right), \\
\Pi_{A B}^{\psi^{r}} & =\left(1-(1-r)^{n+1}\right) v-\pi_{A B}^{r}
\end{aligned}
$$

From the first order condition and Lemma 4.13, the optimal reserve price $r_{A B}^{*}$ maximizing the expected profit of the buyer satisfies

$$
v-r=r-s^{r}
$$




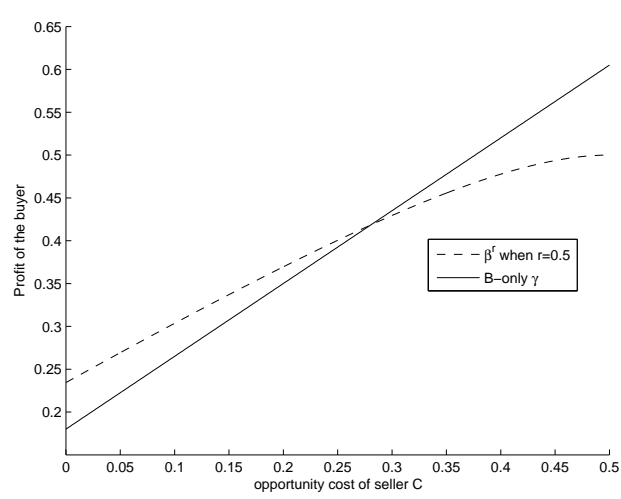

(a) $r=0.5$

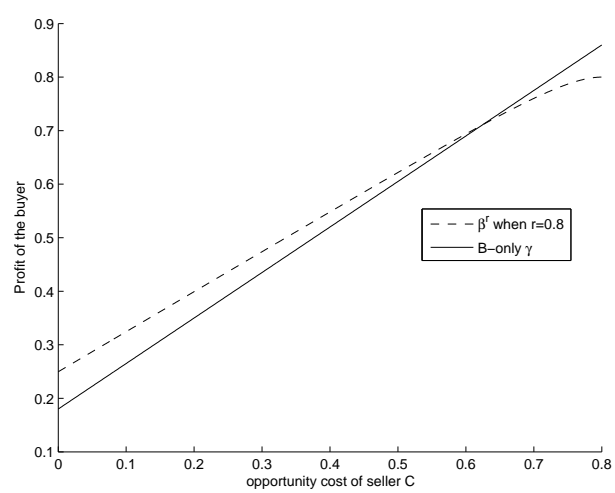

(b) $r=0.8$

Figure 3: The bidding strategy of a seller in the auction-only model with reserve price $\left(\beta^{r}\right)$ and the pricing outcome function (B-only $\gamma$ ) with $\lambda=0.85, v=1.2$, and $n=3$, i.e., 4 sellers.

where $s^{r}$ is the unique intersection between $\beta^{r}$ and $\gamma$ in $[0, r]$. Since $v-r$ is nonnegative, it follows that $r \geq s^{r}$. Thus,

$$
r_{A B}^{*}=\frac{v+s_{A B}^{r_{A B}}}{2}
$$

In the auction-only model, the optimal reserve price for the uniform $[0,1]$ example is given by $r_{A}^{*}=v / 2$ (Lemma 3.2). Thus, it follows that $r_{A B}^{*} \geq r_{A}^{*}$ from the above equation. Thus, the optimal reserve price in the A-B model is less aggressive than in the auction-only model. This observation is consistent with the remark following Lemma 4.13.

Figure 4 is a numerical example of the expected profit of the buyer in the A-B model as a function of the reserve price. Clearly, the buyer's maximum expected profit in the first price A-B model with a reserve price is higher than in the auction-only model with the same reserve price. For the auction-only model, the optimal reserve price is given by $r_{A}^{*}=v / 2=0.6$; for the A-B model, the optimal reserve price is given by $r_{A B}^{*}=(v+s) / 2 \sim 0.95$ where $s$ is numerically calculated to be approximately 0.7 in this case. Thus, we observe that $r_{A B}^{*}$ is bigger than $r_{A}^{*}$. We also observe that for the low values of $r$, the expected profit to the buyers are the same in both models. This occurs since a sufficiently small reserve price $r$ violates the condition $\gamma(0) \leq \beta^{r}(0)$, implying that both $\psi^{r}$ and $\beta^{r}$ functions coincide; thus, the A-B model essentially becomes the auction-only model.

\subsection{Second Price A-B Model}

In the second price A-B model, the seller with the lowest bid in the first phase (auction phase) is selected for the second phase of bargaining, just as in the first price A-B model. 


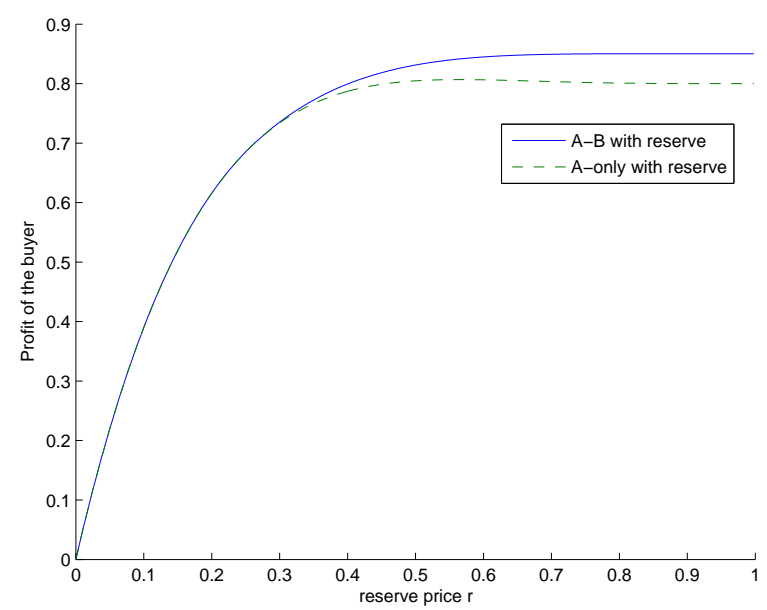

Figure 4: The expected profit of the buyer in the auction-only model with reserve price (A-only) and the first price A-B model (A-B) with reserve price $r$ with $\lambda=0.85, v=1.2$, and $n=3$ (i.e., 4 sellers).

However, the price at which the buyer can purchase the product from the winning bidder is not his bid (the lowest bid), but the second lowest bid. The second price A-B model can easily be implemented if the auction phase is conducted as an open descending-price auction. In this section, we investigate an equilibrium bidding strategy for the first phase in the second price A-B model. We also compare the expected profit of the buyer of the first and second A-B models, and study if a preference ranking between the first and second price A-B models can be established.

In this section, let $\psi(c)$ be a symmetric equilibrium bidding strategy of the second price A-B model. A symmetric equilibrium bidding strategy in the second price A-B model is given in Theorem 4.16.

Theorem 4.16. In the second price A-B model, a dominant bidding strategy for each bidder in the first phase is

$$
\psi(c)=c .
$$

The proof of Theorem 4.16 is similar to the case of the second price auction-only model, and is based on an observation that the amount of payment a bidder expects to receive conditioned on his winning does not depend on his first phase bid. Note that the above strategy is the same as the second price auction-only model, and does not depend on the number of competing sellers in the system. 
The celebrated Revenue Equivalence Theorem implies that the expected profit of the buyer in the first price auction-only model is the same as the corresponding quantity in the second price auction-only model. This theorem is not applicable in the A-B model which is not a mechanism. Now, we compare the expected profits of the buyer, and it turns out that they are generally not identical.

Theorem 4.17. The expected profit of the buyer in the second price $A-B$ model is at least that of the first price $A-B$ model with $\psi(c)=\min \{\beta(c), \gamma(c)\}$.

The proof of Theorem 4.17 is based on comparing the ex post expected payment received by each seller in each of the two models. Let us first review an analogous result in the auctiononly model. It is well known that while the mean of the final price is the same in both the first price auction-only model and the second price auction-only model, the distribution of the final price in the latter model is a mean-preserving spread of the final price in the former model (e.g. Krishna [26]). In the A-B model, a similar analysis shows that the ex post final price in the second price A-B model is a "spread" of the ex post final price in the first-price A-B model; however, the minimum operator in the definition of $\psi$ introduces concavity in taking the expectation over the "spread". Then, by Jensen's Inequality, the expected final price is lower in the second price A-B model, as stated in Theorem 4.17.

\section{Example: Uniform $[0,1]$ Cost in the Second Price A-B Model.}

In the second price A-B model, if the opportunity cost is drawn from uniform $[0,1]$ distribution, the expected profit of the buyer, denoted by $\Pi_{A B}^{2}$, is

$$
\begin{aligned}
\Pi_{A B}^{2} & =v-\sum_{i=1}^{n+1} \mathbb{E}\left[\mathbb{E}\left[\bar{G}(c) \cdot \mathbb{E}[\min \{Y, \gamma(c)\} \mid Y>c] \mid C_{i}=c\right]\right] \\
& =v-\frac{2}{n+2}+\frac{[1-(1-\lambda) v]^{n+2}-\left[(1-\lambda-(1-\lambda) v]^{n+2}\right.}{(n+2) \lambda},
\end{aligned}
$$

where the last equality results from algebraic manipulation. It can be shown from Lemma 3.1 that $\Pi_{A}=v-2 /(n+2)$. Since the last term above is nonnegative, it follows that $\Pi_{A B}^{2} \geq \Pi_{A}$.

Figure 5 shows the expected profit of the buyer in the auction-only, first price A-B, and second price A-B models. We observe that the buyer's expected profit is the highest in the second price A-B model. 


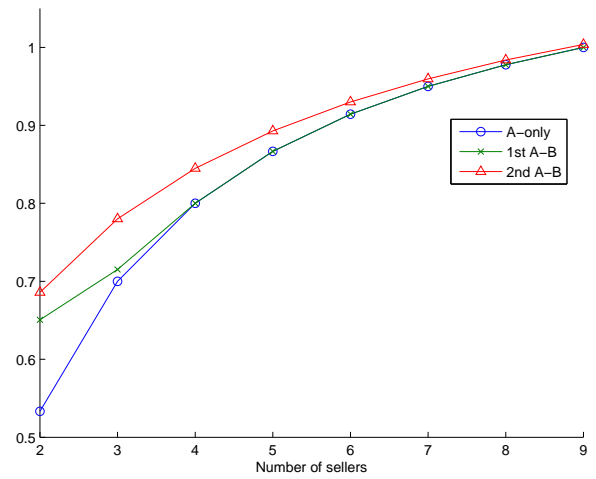

(a) $\lambda=0.75$

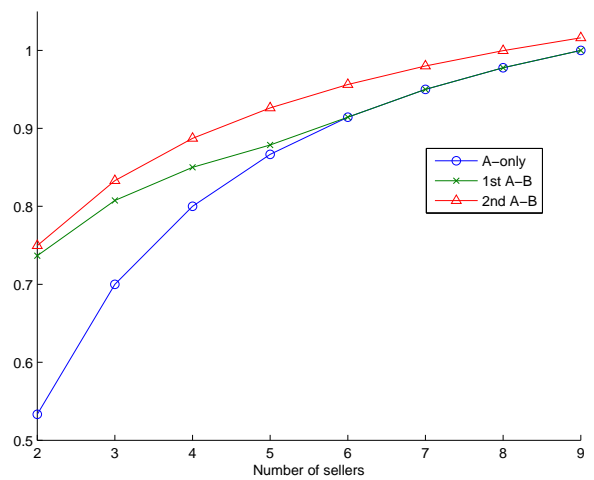

(b) $\lambda=0.85$

Figure 5: The expected profit of the buyer as a function of the number of sellers in the auction-only (A-only), first price A-B (1st A-B), and second price A-B (2nd A-B) models. $v=1.2$.

\section{Extensions and Further Discussions}

In this section, we consider extensions of the models discussed in Sections 3 and 4. In Section 5.1, we first re-visit the auction-bargaining model of Section 4. While only one seller was selected in the auction phase for bargaining in the second phase, in Section 5.1, we consider the case where the buyer can select more than one buyer from the first phase. In Section 5.2 , we discuss the impact of positive fixed costs for conducting an auction or bargaining. In Section 5.3, we consider the sequential bargaining model of Section 3.2 by assuming that the buyer discovers the seller's cost one at a time, and is not allowed to resume bargaining with a seller that she had previously aborted.

\subsection{Multiple Sellers in the Bargaining Phase of the A-B Model}

In the A-B models studied in Section 4, the buyer selects only one seller in the auction phase and enters the second phase bargaining with this seller. In this section, suppose that the buyer selects multiple sellers in the first phase and conducts a sequential bilateral bargaining with these sellers. At the end of the first phase, the buyer observes all the bids $b_{i}$ submitted by sellers $i$, and selects the bidders with the $m$ lowest bids, where $m \in\{2,3, \ldots, n+1\}$. (For notational convenience, suppose that bidders $i=1$ through $m$ are selected for the second phase.) For the second phase bargaining process, the buyer decides the order in which she will sequentially bargain with the $m$ sellers. At the beginning of the second phase, the seller knows the opportunity $\operatorname{costs} c_{i}$ for all sellers $i=1, \ldots, m$. (The second phase bargaining 
with $m$ sellers follows the sequential bargaining model described in Section 3.2.) We refer to this model as Model $M$.

We find an equilibrium bidding strategy of sellers in the first phase, and the bargaining strategy of the buyer in the second phase. Consider the buyer's problem. By an argument similar to the proof of Lemma 3.4, the buyer's payment is independent of the bargaining order, and is given by $\min \left\{\min \left\{b_{i}, \gamma\left(c_{i}\right)\right\} \mid i=1, \ldots, m\right\}$. Thus, one of the buyer's weakly dominant strategies is to bargain first with the seller with the least $\min \left\{b_{i}, \gamma\left(c_{i}\right)\right\}$ (with the least bid) until an agreement is reached. For the sellers' problem, the first-phase bidding strategy can be shown to remain unchanged from Theorem 4.2 of Section 4.

Theorem 5.1. Let $\psi$ be a strictly increasing function defined on $[\underline{c}, \bar{c}]$ by $\psi(c)=\min \{\beta(c), \gamma(c)\}$. If $\psi$ satisfies Condition A, an equilibrium bidding strategy in Model $M$ is as follows: (i) in the first phase, seller $i$ submits the bid of $\psi\left(c_{i}\right)$, (ii) in the second phase, the buyer bargains with the seller with the least $\min \left\{b_{i}, \gamma\left(c_{i}\right)\right\}$, and reaches an agreement with him.

We consider a variation of Model $M$. During the second phase, the seller does not know the ex post $\operatorname{cost} c_{i}$ of a seller $i$ until she enters a round of bilateral bargaining with the seller. Thus, the buyer cannot use $\left\{c_{i} \mid i=1, \ldots, m\right\}$ in deciding the order of bargaining. This is similar to the sequential bargaining model of Section 5.3. For this model, we can establish a result similar to Theorem 5.1, except that the buyer's strategy is to bargain with the seller with the least $b_{i}$ until an agreement is reached.

\subsection{Fixed Costs of Auction and Bargaining}

In our discussion in Section 4, we have assumed that the cost of conducting a bilateral bargaining or auction is negligible. Such an assumption is valid, for example, when there is a good infrastructure for conducting auction or bargaining transactions, or when the buyer's relationship with the sellers have been well maintained such that the auction or bargaining outcomes can be reached without much effort.

In this section, we consider the case where there are positive fixed costs for auction and bargaining, denoted by $K_{A}$ and $K_{B}$, respectively. Then, in the auction-only model, the buyer incurs the cost of $K_{A}$, and in the sequential bargaining model, the buyer incurs the cost of at least one $K_{B}$ (see the discussion in Section 5.3). In the A-B model, the buyer incurs the fixed costs associated with the auction and one round of bargaining, i.e., $K_{A}+K_{B}$. By 
comparing these fixed costs with the expected profits of the buyer in each of these models, the buyer can select the procurement system that maximizes her expected profit.

\subsection{Sequential Bargaining and Optimal Stopping}

We consider the sequential bargaining model where the buyer enters a series of bilateral bargaining with each of $n+1$ potential sellers in an exogenously determined order. Without loss of generality, we assume that this order is given by $i=1,2, \ldots, n+1$. In Section 3.2, we have assumed that the buyer knows the ex post cost $c_{i}$ of each seller $i$, and it has been shown that the buyer's optimal choice is to purchase from the most efficient seller by paying $\gamma\left(\min _{i} c_{i}\right)$. In this section, we assume that the buyer does not know the realized cost $c_{i}$ of seller $i$ until she starts a bargaining process with this seller. Furthermore, the buyer cannot resume bargaining with a seller once she starts bargaining with another seller, i.e., the buyer cannot go back to a previous seller.

We note that this model is an alternative to the A-B model discussed in Sections 3 and 4. In both models, the buyer does not know the seller's cost, unless she initiates bargaining with that seller. In the A-B model, the buyer uses an auction to select one seller with whom she bargains; in the sequential bargaining model discussed here, the buyer does not use any auction, but uses a series of bargaining rounds. In this subsection, we outline a basic analysis for the sequential bargaining model.

We assume that a seller's opportunity cost is independently drawn from a common distribution $F$. In this case, we show that the buyer's profit-maximizing decision strategy of whether to accept a bargaining outcome with each seller is an optimal stopping problem. The following lemma for the optimal stopping criterion follows from Ferguson [21].

Lemma 5.2. In the sequential bargaining model without the buyer's a priori knowledge of $c_{i}$ 's, the optimal stopping criterion for the buyer is to accept the bargaining outcome with the j'th seller if $c_{j} \leq A_{j}$ where

$$
A_{j}= \begin{cases}\infty, & j=n+1 \\ \mathbb{E}\left[\min \left\{C_{j+1}, A_{j+1}\right\}\right], & j \in\{n, n-1, \ldots, 1\} .\end{cases}
$$

Furthermore, if $C_{i}$ is uniformly distributed on $[0,1]$, then $A_{n}=1 / 2$ and $A_{j}=A_{j+1}-A_{j+1}^{2} / 2$ for $j=n-1, \ldots, 1$.

Given the above decision rule of the buyer, seller $j$ will choose to sell to the buyer at the price of $\gamma\left(c_{j}\right)$ if $c_{j} \leq A_{j}$. If $A_{j}<c_{j} \leq \gamma\left(A_{j}\right)$, then he will procure and receive $\gamma\left(A_{j}\right)$ instead 
since it is still profitable to do so. Otherwise, he will not reach an agreement with the buyer and the buyer will start bargaining with the next seller $j+1$.

We can easily extend the above analysis to the case where the buyer incurs a fixed cost $K_{B}$ for each round of bargaining. This cost is incurred, for example, when the buyer researches a seller's ex post cost at the beginning of bargaining with the seller. Then, it can be shown that the optimal stopping criterion of Lemma 5.2 remains valid except for the following modification: for $j \in\{n, n-1, \ldots, 1\}$,

$$
A_{j}=\mathbb{E}\left[\min \left\{C_{j+1}, A_{j+1}-K_{B} / \lambda\right\}\right]
$$

(Note that $1-\lambda$ is the bargaining power of the seller.)

In the special case where the buyer knows that the first seller is the most efficient seller, i.e., $c_{1}=\min _{i} c_{i}$, then it is optimal for the buyer to reach an agreement with the first seller. In this case, the buyer pays $\gamma\left(c_{1}\right)=\min _{i} \gamma\left(c_{i}\right)$.

\section{Conclusion}

In this paper, we have examined a combined auction-bargaining model in a procurement setting, where the buyer procures an indivisible item from one of many competing sellers. The model consists of two phases: in the auction phase, the buyer selects the seller, and in the bargaining phase, the final price is determined. The winning bid in the auction phase serves as an outside option for the buyer in the bargaining phase. As a result, each seller's bidding strategy in the auction phase strikes a balance between increasing his probability of winning, and increasing the final price in case that he wins. In this paper, we take the perspective of the expected profit maximizing buyer, and allow the buyer to set a reserve price in the first phase.

For our model, we find a symmetric first-phase bidding strategy for the sellers, which is simple and intuitive to understand. We show that the combined auction-bargaining model produces a higher expected profit to the buyer than the standard auction or sequential bargaining models. The buyer's expected profit can be improved by setting an appropriate reserve price in the auction phase, and in many cases, the optimal reserve price can easily be computed from the first order condition. We also show that the buyer prefers conducting the auction phase with the first price auction to the second price auction. Our results are illustrated using a uniform distribution example. 
We believe that there are several interesting extensions that can be addressed in the framework of the auction-bargaining model proposed above. For example, when the sellers are asymmetric, both in terms of the distribution of cost and the bargaining power, it would be interesting to investigate what kind of sellers would benefit from the auction-bargaining model as opposed to the standard auction or bargaining.

\section{Acknowledgement}

The authors are grateful for the suggestions of Yeon-Koo Che and Mahesh Nagarajan. The results and exposition of this paper have been improved by comments by the Associate Editor and two anonymous referees.

\section{References}

[1] P. L. Bajari, R. S. McMillan, and S. Tadelis, Auctions versus negotiations in procurement: An empirical analysis, forthcoming to Journal of Law, Economics, and Organization, 2008.

[2] D. R. Beil, and L. M. Wein, An inverse-optimization-based auction mechanism to support a multiattribute RFQ process, Management Science 49 (2003), 1529-1545.

[3] S. Benjaafar, E. Elahi, and K. L. Donohue, Outsourcing via service competition, Management Science 53 (2007), 241-259.

[4] K. Binmore, "Bargaining and coalitions", Game Theoretic Models of Bargaining, A. E. Roth (Editors), Cambridge University Press, 1985, pp. 269-304.

[5] A. Bonaccorsi, P. L. Thomas, F. Pammolli, and G. Turchetti, Auctions vs. bargaining: An empirical analysis of medical device procurement, Laboratory of Economics and Management Papers Series 1999/20, Sant'Anna School of Advanced Studies, Pisa, Italy, 1999.

[6] J. S. Bonser, and S. D. and Wu, Procurement planning to maintain both short-term adaptiveness and long-term perspective, Management Science 47 (2001), 769-786.

[7] E. B. Budish, and L.N. Takeyama, Buy prices in online auctions: Irrationality on the internet?, Economic Letters 72 (2001), 325-333. 
[8] J. Bulow, and P. Klemperer, Auctions versus negotiations, The American Economic Review 86 (1996), 180-194.

[9] G. P. Cachon, and F. Zhang, Procuring fast delivery: Sole sourcing with information asymmetry, Management Science 52 (2006), 881-896.

[10] R. Caldentey, and G. Vulcano, Online auctions and list price revenue management, Management Science 53 (2007), 795-813.

[11] Y.-K. Che, Design competition through multidimensional auctions, The RAND Journal of Economics 24 (1993), 668-680.

[12] Y.-K. Che, The effect of defense profit policy on quality determination, Economics Letters 45 (1994), 397-405.

[13] F. Chen, Auctioning supply contracts, Management Science 53 (2007), 1562-1576.

[14] R. R., Chen, R. O. Roundy, R. Q. Zhang, and G. Janakiraman, Efficient auction mechanisms for supply chain procurement, Management Science 51 (2005), 467-482.

[15] S. P., Dalya, and P. Nath, Online reverse auctions: Issues, themes, and prospects for the future, Industrial Marketing Management 34 (2005), 157-166.

[16] W. Elmaghraby, P. Keskinocak, and M. Rothkopf, Introduction to the special issue dynamic pricing policies in electronic commerce, Electronic Commerce Research Journal 5 (2005), 175-180.

[17] B. Elyakime, J. Laffont, P. Loisel, and Q. Vuong, Auctioning and bargaining: An econometric study of timber auctions with secret reservation prices, Journal of Business and Economic Statistics 15 (1997), 209-220.

[18] R. Engelbrecht-Wiggans, and E. Katok, E-sourcing in procurement: Theory and behavior in reverse auctions with noncompetitive contracts, Management Science 52 (2006), $581-596$.

[19] P. Eső, and B. Szentes, Optimal information disclosure in auctions and the handicap auction, Review of Economic Studies 74 (2007), 705-731. 
[20] A. Federgruen, and A. Heching, Combined pricing and inventory control under uncertainty, Operations Research 47 (1999), 454-475.

[21] T. S. Ferguson, Optimal Stopping and Applications, Mathematics Department, UCLA, 2000 ,

URL http://www. math.ucla.edu/ tom/Stopping/Contents.html.

[22] J. Gallien, and S. Gupta, Temporary and permanent buyout prices in online auctions, Management Science 53 (2007), 814-833.

[23] Z. Hidvégi, W. Wang, and A.B. Whinston, Buy-price english auction, Journal of Economic Theory 129 (2006), 31-56.

[24] S. D. Jap, Online reverse auctions: Issues, themes, and prospects for the future, Journal of the Academy of Marketing Science 30 (2002), 506-525.

[25] E. Kjerstad, Auctions vs negotiations: A study of price differences, Health Economics 14 (2005), 1239-1251.

[26] V. Krishna, Auction Theory, Academy Press, 2002.

[27] K. B. Leffler, and T. R. Rucker, I. A. Munn, The choice among sales procedures: Auction vs. negotiated sales of private timber. Working Paper, Department of Economics, University of Washington, 2003.

[28] A. M. Manelli, and D. R. Vincent, Optimal procurement mechanisms, Econometrica 63 (1995), 591-620.

[29] T. Mathews, The impact of discounting on an auction with a buyout option: a theoretical analysis motivated by ebay's buy-it-now feature, Journal of Economics 81 (2004), $24-52$.

[30] F. M. Menezes, and P. K. Monteiro, Introduction to Auction Theory, Oxford University Press, 2005.

[31] A. Muthoo, Bargaining Theory with Applications, Cambridge University Press, 1999.

[32] R. Myerson, Optimal auction design, Mathematics of Operations Research 6 (1981), $58-73$. 
[33] M. Nagarajan, and Y. Bassok, A bargaining framework in supply chains, Management Science 54 (2008), 1482-1496.

[34] M. Nagarajan, and G. Sosic, Game-theoretic analysis of cooperation among supply chain agents: Review and extensions, European Journal of Operational Research 187 (2008), 719-745.

[35] M. Osborne, and A. Rubinstein, Bargaining and Markets, Academy Press, 1990.

[36] A. Rubinstein, Perfect equilibrium in a bargaining model, Econometrica 50 (1982), 97109.

[37] T. C. Salmon, and B. J. Wilson, Second chance offers versus sequential auctions: Theory and behavior. Economic Theory 34 (2008), 47-67.

[38] A. Shaked, Opting-out: Bazaars versus "high-tech" markets, Investigations Economics 18 (1994), 421-432.

[39] C. J. Thomas, and B. J. Wilson, A comparison of auctions and multilateral negotiations, RAND Journal of Economics 33 (2002), 140-155.

[40] T. I. Tunca, and S. A. Zenios, Supply auctions and relational contracts for procurement, Manufacturing and Service Operations Management 8 (2006), 43-67.

[41] K. Waehrer, The ratchet effect and bargaining power in a two-state model of competive bidding, Economic Theory 13 (1999) 171-181.

[42] Z. Wan, and D. R. Beil, RFQ auctions with supplier qualification screening, forthcoming to Operations Research, 2008.

[43] R. Wang, Bidding and renegotiation in procurement auctions, European Economic Review 15 (2000), 1577-1597.

[44] Wu, S. D., "Supply chain intermediation: A bargaining theoretic framework", Handbook of Quantitative Supply Chain Analysis in the eBusiness Era, D. Simchi-Levi, S.D. Wu, M. Shen (Editors), 2004, Kluwer Academic Publishers, pp. 67-115.

[45] L. Ye, Indicative bidding and a theory of two-stage auctions, Games and Economic Behavior 58 (2007), 181-207. 


\section{A. Proof of Lemma 4.1}

Proof. We prove $\psi(c) \leq \gamma(c)$. Suppose, by way of contradiction, $\psi(c)>\gamma(c)$. Suppose a seller has the ex post cost $c$ and places a bid of $\psi(c)$ in the auction phase of the A-B model. If he becomes the winning bidder, then the final price outcome in the second-phase bargaining process will be $\gamma(c)$ since $\gamma(c)<\psi(c)$. Since the probability density function $f$ is strictly positive at $c \in(\underline{c}, \bar{c})$, by perturbing his bid lower, the seller strictly increases the probability of his winning without affecting the outcome of the final price in the scenario in which he wins the auction phase. Thus, bidding more than $\gamma(c)$ in the auction phase is not optimal for the seller.

\section{B. Proof of Theorem 4.2}

Proof. To show that $\psi(c)=\min \{\beta(c), \gamma(c)\}$ is a symmetric equilibrium, we suppose that all the other sellers except seller $i \in\{1,2, \ldots, n+1\}$ follow this strategy, and show that seller $i$ achieves the maximum expected profit by also following $\psi$. Suppose $c \in[\underline{c}, \bar{c}]$ is the ex post cost of bidder $i$, and let $b$ be his first-phase bid. By Lemma 4.1, $b \leq \gamma(c)$. Also, it is clearly not optimal for seller $i$ to place a bid lower than $\psi(\underline{c})$. Thus, $\psi(\underline{c}) \leq b \leq \gamma(c)$.

Let $z$ be defined by $\psi(z)=b$. Since $\psi$ is a strictly increasing and continuous function, $z$ is well-defined. We refer to $z$ as the implied cost associated with his bid $b$. From the above argument, $\psi(\underline{c}) \leq \psi(z) \leq \gamma(c)$. Then, seller $i$ 's expected profit is given by the probability of winning multiplied by his expected profit, conditioned on his winning the auction. The probability of winning the auction phase is $\bar{G}(z)$. If seller $i$ wins the auction phase, then the price outcome of the bargaining phase is the minimum of his bid $\psi(z)$ and $\gamma(c)$, i.e., $\min \{\psi(z), \gamma(c)\}$ which is $\psi(z)$. Thus, seller $i$ 's expected profit is

$$
\Pi_{i}^{\psi}(z, c)=\bar{G}(z)(\psi(z)-c)=\bar{G}(z)(\min \{\beta(z), \gamma(z)\}-c)
$$

We prove that, if $\psi$ satisfies Condition $\mathrm{A}, \Pi_{i}^{\psi}(z, c)$ is maximized at $z=c$ by showing that $\Pi_{i}(z, c)$ increases in $z$ if $z<c$ and decreases in $z$ if $z>c$. We consider the following three cases.

Case $\beta(z)<\gamma(z)$ : Since $\beta$ and $\gamma$ intersect finitely many times, there exists $\epsilon>0$ such that $\beta\left(z^{\prime}\right)<\gamma\left(z^{\prime}\right)$ for any $z^{\prime} \in(z-\epsilon, z+\epsilon)$. Thus, it follows $\psi\left(z^{\prime}\right)=\beta\left(z^{\prime}\right)$ and $\Pi_{i}\left(z^{\prime}, c\right)=\bar{G}\left(z^{\prime}\right)\left(\beta\left(z^{\prime}\right)-c\right)$. Differentiating $\Pi_{i}^{\psi}(z, c)$ with respect to $z$ in $(z-\epsilon, z+\epsilon)$ when 
$\psi(z)=\beta(z)$, we obtain

$$
\frac{\partial}{\partial z} \Pi_{i}^{\psi}(z, c)=-g(z)(\beta(z)-c)+\bar{G}(z) \frac{\partial}{\partial z} \beta(z)
$$

Since $\beta(z)=\mathbb{E}[Y \mid Y>z]$ follows from Lemma 3.1, it is straightforward to verify

$$
\frac{\partial}{\partial z} \beta(z)=\frac{g(z)}{\bar{G}(z)}(\beta(z)-z) .
$$

Combining these two equations,

$$
\frac{\partial}{\partial z} \Pi_{i}^{\psi}(z, c)=g(z)(c-z)
$$

If $z<c$, then the above expression is positive, and perturbing $z$ higher increases the expected profit. Otherwise, perturbing $z$ lower increases the expected profit.

Case $\gamma(z)<\beta(z)$ : As before, there exists $\epsilon>0$ such that $\gamma\left(z^{\prime}\right)<\beta\left(z^{\prime}\right)$ for any $z^{\prime} \epsilon$ $(z-\epsilon, z+\epsilon)$. Differentiating the expected profit with respect to $z$ when $\psi(z)=\gamma(z)$, we obtain

$$
\frac{\partial}{\partial z} \Pi_{i}^{\psi}(z, c)=-g(z)(\gamma(z)-c)+\bar{G}(z) \lambda
$$

Note that by Lemma 4.1, we obtain $\gamma(z)=\psi(z) \leq \gamma(c)$, which implies $z \leq c$. If $z<c$, then $\psi(z)=\gamma(z)$ and Condition A together imply that (4) is nonnegative since

$$
\lambda \geq \frac{g(z)}{\bar{G}(z)}(\gamma(z)-z) \geq \frac{g(z)}{\bar{G}(z)}(\gamma(z)-c),
$$

where the first inequality follows from Condition A and the second inequality follows from $z<c$. Thus, perturbing $z$ higher weakly increases the expected profit.

Case $\gamma(z)=\beta(z)$ : There exists $\epsilon>0$ such that either $\beta\left(z^{\prime}\right)<\gamma\left(z^{\prime}\right)$ or $\beta\left(z^{\prime}\right)>\gamma\left(z^{\prime}\right)$ for either $z^{\prime} \in(z, z+\epsilon)$ or $z^{\prime} \in(z-\epsilon, z)$. Then, apply the argument used in one of the two cases discussed above accordingly.

If Condition A does not hold, then there exists $c$ such that $\psi(c)=\gamma(c)<\beta(c)$ and $\left.\frac{\partial}{\partial z} \Pi_{i}^{\psi}(z, c)\right|_{z=c}<0$; thus, bidding lower than $\gamma(c)$ improves the profit. Thus $\psi$ is not an equilibrium bidding strategy. (See the proof of Lemma 4.6 for the detail.) 


\section{Proof of Lemma 4.3}

Proof. To prove the lemma, suppose $\gamma^{\prime}(c)=\lambda \geq \beta^{\prime}(c)$ for any $c \in[\underline{c}, \bar{c}]$. Recall $\psi(c)=$ $\min \{\gamma(c), \beta(c)\}$. Since $\psi(c)=\gamma(c)$ implies $\gamma(c) \leq \beta(c)$,

$$
\frac{g(c)}{\bar{G}(c)}(\gamma(c)-c) \leq \frac{g(c)}{\bar{G}(c)}(\beta(c)-c)=\frac{\partial}{\partial c} \beta(c),
$$

where the equality follows from the definition of $\beta$ (see (3)). Thus, from $\beta^{\prime}(c) \leq \lambda$, we obtain (1), completing the proof.

\section{Proof of Theorem 4.5}

Proof. Let $\psi$ be a continuous increasing function defined on $[\underline{c}, \bar{c}]$. First, if $\psi$ satisfies both (i) and (ii), then an argument similar to the proof of Theorem 4.2 shows that $\psi$ is an equilibrium.

Suppose now $\psi$ is a symmetric equilibrium function. We show that $\psi$ satisfies (i) and (ii). By Lemma 4.1, we obtain $\psi(c) \leq \gamma(c)$ for each $c \in[\underline{c}, \bar{c}]$, proving (i). For (ii), let $c^{\circ} \in(\underline{c}, \bar{c})$ such that $\psi\left(c^{\circ}\right)<\gamma\left(c^{\circ}\right)$. Let $s=\sup \left\{c<c^{\circ} \mid \psi(c)=\gamma(c)\right.$ or $\left.c=\underline{c}\right\}$ and $t=\inf \left\{c>c^{\circ} \mid \psi(c)=\gamma(c)\right.$ or $\left.c=\bar{c}\right\}$. By the continuity of both $\psi$ and $\gamma$, it follows $c^{\circ} \in(s, t)$. Let $\Pi_{i}(z, c)$ be the seller $i$ 's expected profit when seller $i$ submits the bid of $\psi(z)$ when his cost is $c$, and all other bidders follow the bidding strategy $\psi$. Since $\psi$ is an equilibrium, $\Pi_{i}(z, c)$ for fixed $c$ is maximized when $z=c$. Differentiating $\Pi_{i}(z, c)=\bar{G}(z)(\psi(z)-c)$ with respect to $z$ in $(s, t)$, we obtain

$$
\frac{\partial}{\partial z} \Pi_{i}(z, c)=-g(z)(\psi(z)-c)+\bar{G}(z) \frac{\partial}{\partial z} \psi(z) .
$$

It is straightforward to verify that the family of solutions for $\psi$ satisfying the above differential equation to be 0 at $z=c$ is

$$
\beta_{K}(c)=\mathbb{E}[Y \mid Y>c]+\frac{K}{\bar{G}(c)},
$$

parameterized by $K$. The choice of $K$ is unique by the boundary condition at $c^{\circ}$, namely $\beta_{K}\left(c^{\circ}\right)=\psi\left(c^{\circ}\right)$. Thus, $\psi(c)=\beta_{K}(c)$ for $c \in[s, t]$. Thereby, we verify (ii).

Furthermore, we prove the property of $\psi$ in the neighborhood of $\bar{c}$. Recall from Section 2 our assumption $v \geq \bar{c}$, which implies $\gamma(\bar{c}) \geq \beta_{0}(\bar{c})=\bar{c}$. By the first part of Theorem 4.5 , in the interval $\left[a_{m-1}, a_{m}\right]$ where $a_{m}=\bar{c}$, we have either $\psi(c)=\beta_{K}(c)$ for some $K$, or $\psi(c)=\gamma(c)$. In the former case, $K$ cannot be positive since $\beta_{K}(c)$ should be well-defined 
and finite for all $c$ in the closed interval $\left[a_{m-1}, a_{m}\right]$. Also, $K$ cannot be negative since $\beta_{K}(c)$ should be increasing in the neighborhood of $\bar{c}$. Thus, we only need to consider $\beta_{0}$ and $\gamma$.

If $\gamma(\bar{c})=\beta_{0}(\bar{c})$, then the above result shows that $\psi(\bar{c})=\gamma(\bar{c})=\beta_{0}(\bar{c})=\bar{c}$. We proceed with the case $\gamma(\bar{c})>\beta_{0}(\bar{c})$, and will prove $\psi(c)=\beta_{0}(c)$ in $\left[a_{m-1}, a_{m}\right]$. Suppose by the way of contradiction that $\psi(c)=\gamma(c)$ in this interval. Then, since $g(c)=n \cdot(1-F(c))^{n-1} f(c)$ and $\bar{G}(c)=(1-F(c))^{n}$, we have

$$
\frac{g(c)}{\bar{G}(c)}(\gamma(c)-c)=n \cdot \frac{f(c)}{1-F(c)}(\gamma(c)-c) .
$$

By the assumption that $f(c)>0$ for all $c \in[\underline{c}, \bar{c}]$ where $\bar{c}<\infty$ (from Section 2), it follows that the above expression can be arbitrarily large as $c \rightarrow \bar{c}$. In particular, the above expression exceeds $\lambda$, which violates Condition A. Thus, we conclude $\psi(c)=\beta_{0}(c)$ in $\left[a_{m-1}, a_{m}\right]$.

\section{E. Proof of Lemma 4.6}

Proof. We prove the lemma by contradiction. Suppose there exists $c$ in the interior of $\Gamma^{\psi}$ such that Inequality (1) does not hold, i.e., $\psi(c)=\gamma(c)$ and

$$
\lambda<\frac{g(c)}{\bar{G}(c)}(\gamma(c)-c) \text {. }
$$

It follows that there exists $\epsilon>0$ such that $(c-\epsilon, c) \subseteq \Gamma^{\psi}$, and the above inequality still holds in this interval. Suppose that all the other bidders follow the bidding strategy $\psi$. We proceed to show that seller $i$ 's expected profit is higher when he bids $\psi(c-\epsilon)$ compared to $\psi(c)$, which violates the definition of an equilibrium.

Differentiating the expected profit of seller $i$ with respect $z \in(c-\epsilon, c)$, we obtain from $(4)$,

$$
\begin{aligned}
\frac{\partial}{\partial z} \Pi_{i}^{\psi}(z, c) & =-g(z)(\gamma(z)-c)+\bar{G}(z) \lambda \\
& =\bar{G}(z) \cdot\left(-\frac{g(z)}{\bar{G}(z)}(\gamma(z)-c)+\lambda\right)
\end{aligned}
$$

which is negative by the choice of $\epsilon$ above. Thus, $\Pi_{i}(c-\epsilon, c)<\Pi_{i}(c, c)$, and it follows that $\psi$ is not an equilibrium strategy. 


\section{F. Proof of Theorem 4.9}

Proof. From Lemma 4.1, recall $\psi(c) \leq \gamma(c)$ for any $c \in(\underline{c}, \bar{c})$. Thus, if we can prove $\psi(c) \leq \beta(c)$ for $c \in(\underline{c}, \bar{c})$, then we obtain $\psi(c) \leq \min \{\beta(c), \gamma(c)\}$, which implies the required result.

Therefore, for the remainder of the proof, we prove $\psi(c) \leq \beta(c)$ for $c \in(\underline{c}, \bar{c})$. Consider the characterization of $\psi(c)$ given in the statement of Theorem 4.5. From the discussion following Lemma 4.6, we have $\psi(\bar{c})=\beta_{0}(\bar{c}) \leq \gamma(\bar{c})$. Let

$$
s_{1}=\min \left\{\tilde{c} \in[\underline{c}, \bar{c}] \mid \beta_{0}(c) \leq \gamma(c) \text { for any } c \in[\tilde{c}, \bar{c}]\right\}
$$

If $s_{1}=\underline{c}$, then we must have $\psi(c)=\beta(c)$, which proves the required result. Otherwise, define

$$
s_{2}=\min \left\{\tilde{c} \in\left[\underline{c}, s_{1}\right] \mid \beta_{0}(c) \geq \gamma(c) \quad \text { for any } \quad c \in\left[\tilde{c}, s_{1}\right]\right\}
$$

Then, from the continuity of $\psi$ and the monotonicity of $\beta_{K}$ in $K$, it follows that, for any $c \in\left[s_{2}, s_{1}\right]$, we have $\psi(c)=\gamma(c)$ or $\psi(c)=\beta_{K}(c)$ for some $K \leq 0$. Therefore, if $s_{2}=\underline{c}$, then the required result holds. However, if $s_{2}>\underline{c}$, then the choice of $s_{2}$ implies $\beta_{0}^{\prime}\left(s_{2}\right)>\gamma^{\prime}\left(s_{2}\right)=\lambda$, which is contrary to Condition A (apply the same logic as the proof of Lemma 4.3). Thus, we conclude that $s_{2}=\underline{c}$.

\section{G. Proof of Theorem 4.10}

Proof. From the remarks following Theorem 4.5, we obtain $\psi(1)=1$. Thus, by applying Theorem 4.5, the right-most segment is the $\beta_{0}$ segment; more precisely, let $s=\inf \{c \in$ $[0,1] \mid \beta_{0}(c) \leq \gamma(c)$ or $\left.c=0\right\}$. Then, $\psi(c)=\beta_{0}(c)$ for all $c \in[s, 1]$. Since $s=0$ implies that

the statement of the theorem holds, we proceed by assuming $s>0$. Then, it is easy to verify that if $K>0$, then $\beta_{K}(c)>\beta_{0}(c)>\gamma(c)$ in $[0, s)$, implying that $\beta_{K}$ and $\gamma$ do not intersect in $[0, s)$; thus, $\beta_{K}$ does not specify any of the segments. If $K<0$, then $\beta_{K}$ crosses $\gamma$ at most once at $[0, s]$, in which case, it crosses from above to below (not from below to above). Then, because of the constraint $\psi(c) \leq \gamma(c)$, we cannot construct a continuous increasing function $\psi$ satisfying $\psi(\tilde{c})=\beta_{K}(\tilde{c})<\gamma(\tilde{c})$ and $\psi(\hat{c})=\beta_{K}(\hat{c})<\gamma(\hat{c})$ where $0 \leq \tilde{c}<\hat{c} \leq 1$. Thus, $\beta_{K}$ does not specify any of the segments. 


\section{H. Uniform[0,1] Cost Example in the First Price A-B Model: Buyer's Expected Profit}

With the Uniform $[0,1]$ cost distribution, the unique equilibrium bidding strategy in the A-B model is given by Theorem 4.10 of Section 4.1. Here, we give a closed-form expression for the buyer's expected profit in the A-B model, and compare it to the other models.

Recall that the buyer's expected profits in the auction-only model and in the sequential bargaining model are given by the following expressions. These results are straightforward to verify.

$$
\begin{aligned}
\Pi_{A} & =v-(n+1) \mathbb{E}[\bar{G}(c) \beta(c)] \\
& =v-(n+1) \int_{0}^{1} \beta(c) \bar{G}(c) f(c) d c=v-\frac{2}{n+2}, \\
\Pi_{B} & =v-(n+1) \mathbb{E}[\bar{G}(c) \gamma(c)] \\
& =v-(n+1) \int_{0}^{1} \gamma(c) \bar{G}(c) f(c) d c=\lambda \cdot v-\frac{\lambda}{n+2} .
\end{aligned}
$$

Note that the ranking between $\Pi_{A}$ and $\Pi_{B}$ depends on the values of $\lambda$ and $v$.

We now compute the expected profit $\Pi_{A B}$ of the buyer in the A-B model. Suppose that the two linear functions $\beta$ and $\gamma$ intersect in $[0,1]$. The intersection point is given by

$$
s=\frac{1-(n+1)(1-\lambda) v}{(n+1) \lambda-n} .
$$

An algebraic manipulation shows

$$
\begin{aligned}
\Pi_{A B}= & v-(n+1) \mathbb{E}[\psi(c) \bar{G}(c)] \\
= & v-(n+1)\left(\int_{0}^{s} \gamma(c) \bar{G}(c) f(c) d c+\int_{s}^{1} \beta(c) \bar{G}(c) f(c) d c\right) \\
= & v-\frac{2}{n+2}+[1-(n+1)(1-\lambda) v] \frac{1-(1-s)^{n+1}}{n+1} \\
& +[(n+1) \lambda-n]\left[\frac{s(1-s)^{n+1}}{n+1}-\frac{1-(1-s)^{n+2}}{(n+1)(n+2)}\right] .
\end{aligned}
$$

\section{Proof of Lemma 4.13}

Proof. To derive optimal reserve price $r_{A B}^{*}$ in the first price A-B model, we calculate the expected profit of the buyer with the reserve price $r$ and maximize her expected profit with 
respect to $r$. The expected profit of the buyer in the first price A-B model with reserve price $r$ is

$$
\Pi_{A B}^{\psi^{r}}=\left(1-(1-F(r))^{n+1}\right) \cdot v-(n+1) \cdot \int_{\underline{c}}^{r} \psi^{r}(c) \bar{G}(c) f(c) d c .
$$

Differentiating the expected profit with respect to $r$ yields,

$$
\frac{\partial}{\partial r} \Pi_{A B}^{\psi^{r}}=(n+1) \cdot \bar{G}(r) \cdot f(r) \cdot(v-r)-(n+1) \cdot \int_{\underline{c}}^{r} \bar{G}(c) \cdot f(c) \cdot \frac{\partial}{\partial r} \psi^{r}(c) d c .
$$

Since $\psi^{r}(c)=\min \left\{\beta^{r}(c), \gamma(c)\right\}$, differentiating $\psi^{r}$ with respect to $r$ gives

$$
\frac{\partial}{\partial r} \psi^{r}(c)= \begin{cases}\frac{\partial}{\partial r} \beta^{r}(c)=\frac{\bar{G}(r)}{\bar{G}(c)} & \text { if } \psi^{r}(c)=\beta^{r}(c) \\ \frac{\partial}{\partial r} \gamma(c)=0 & \text { if } \psi^{r}(c)=\gamma(c)\end{cases}
$$

Substituting this into the above differentiation of the expected profit, we have

$$
\frac{\partial}{\partial r} \Pi_{A B}^{\psi^{r}}=(n+1) \cdot \bar{G}(r) \cdot\left[f(r) \cdot(v-r)-\int_{\underline{c}}^{r} I\left\{\beta^{r}(c) \leq \gamma(c)\right\} \cdot f(c) d c\right] .
$$

Note that the first order condition implies $\partial \Pi_{A B}^{\psi^{r}} / \partial r=0$. Since $r<\bar{c}$ and $\bar{G}(r)$ is strictly positive for any $r \in[\underline{c}, \bar{c})$, we obtain

$$
f(r) \cdot(v-r)-\int_{\underline{c}}^{r} I\left\{\beta^{r}(c) \leq \gamma(c)\right\} \cdot f(c) d c=0
$$

as required.

\section{J. Proof of Theorem 4.17}

Proof. Suppose that seller $i$ 's ex post cost is $c$. We compare the payment received by seller $i$ in the first price and the second price A-B models. Since the first phase bidding functions in Theorems 4.10 and 4.16 are symmetric and increasing, seller $i$ wins the auction phase if $c<C_{j}$ for each $j \neq i$. Otherwise, seller $i$ does not receive any payment from the buyer.

We compare the payments received by seller $i$ given that he wins the auction phase of the A-B model, i.e., $Y>c$. Let $P_{i}^{1}(c, Y)$ and $P_{i}^{2}(c, Y)$ denote these quantities in the first price and the second price A-B models, respectively. (The superscript indicates the first price or second price auction.) Let $\psi^{1}$ and $\psi^{2}$ denote the equilibrium bidding strategy given in Theorems 4.10 and 4.16 , respectively. Then,

$$
\begin{aligned}
& P_{i}^{1}(c, Y)=\min \{\gamma(c), \beta(c)\} \\
& P_{i}^{2}(c, Y)=\min \{\gamma(c), Y\} .
\end{aligned}
$$


Let $m_{i}^{1}(c)$ denote the ex post conditional expected revenue received by seller $i$ when his realized cost is $c$, i.e., the expected value of $P_{i}^{1}(c, Y)$ where the expectation is taken for all values of $Y$ satisfying $Y>c$. Similarly, define $m_{i}^{2}(c)$. Then,

$$
\begin{aligned}
& m_{i}^{1}(c)=\min \{\gamma(c), \beta(c)\}=\min \{\gamma(c), \mathbb{E}[Y \mid Y>c]\} \\
& m_{i}^{2}(c)=\mathbb{E}[\min \{\gamma(c), Y\} \mid Y>c] .
\end{aligned}
$$

Observe that $\min \{\gamma(c), y\}$ is a concave function with respect to $y$. We apply Jensen's Inequality to this function for the conditional distribution $[Y \mid Y>c]$, and obtain $m_{i}^{1}(c) \geq m_{i}^{2}(c)$. Since the ex post expected revenue received by seller $i$ is higher in the first price A-B model, the buyer's expected profit is lower in the A-B model.

\section{K. Proof of Theorem 5.1}

Proof. From the discussion preceding the statement of this theorem, it is easy to verify that the buyer's strategy is the best response to the sellers' bidding strategy, which is symmetric and increasing.

We now consider each seller $i$ 's best response given that the buyer and all the other sellers follow the strategy given in the statement. Suppose that seller $i$ has the cost of $c_{i}$ and bids $b_{i}$ in the first phase. Since $\psi$ is an increasing function, by an argument in the proof of Theorem 4.2, there exists $z$ such that $\psi(z)=b_{i}$ and $\psi(\underline{c}) \leq \psi(z) \leq \gamma\left(c_{i}\right)$. Without loss of generality, suppose that $i=n+1$, and that the other sellers $j \in\{1, \ldots, n\}$ are indexed in an increasing order of $b_{j}$. Since $b_{j}=\psi\left(c_{j}\right)=\min \left\{\beta\left(c_{j}\right), \gamma\left(c_{j}\right)\right\}$ for $j \in\{1, \ldots, n\}$, sellers other than $n+1$ are indexed in an increasing order of $\psi\left(c_{j}\right)$, and also in an increasing order of $c_{j}$.

Thus, seller $i=n+1$ is one of $m$ sellers selected in the first phase if and only if $\min \left\{b_{i}, \gamma\left(c_{i}\right)\right\}<\psi\left(c_{m}\right)$ (assuming no ties). Since $b_{i}=\psi(z) \leq \gamma\left(c_{i}\right)$, this condition is equivalent to $\psi(z)<\psi\left(c_{m}\right)$. Furthermore, seller $i$ wins in the second phase if and only if $b_{i}=\psi(z)<\min \left\{\psi\left(c_{j}\right), \gamma\left(c_{j}\right)\right\}=\psi\left(c_{j}\right)$ for each $j=1, \ldots, m$. In summary, seller $i$ is selected to procure if and only if $z<c_{1}$. In the case that seller $i$ wins, the final price is $\psi(z)$ and his profit is $\psi(z)-c$. Therefore, we obtain (2), and the remainder of the proof is similar to the proof of Theorem 4.2. 